\title{
Gradhiva
}

GRADHIV

Revue d'anthropologie et d'histoire des arts

26 | 2017

En croire ses sens

\section{Architectures divines. Espace et kinesthésie dans le palo monte afro-cubain}

Divine Architectures. Space and Kinaesthetics in Afro-Cuban Palo Monte

\section{Katerina Kerestetzi}

\section{OpenEdition}

1 Journals

Édition électronique

URL : http://journals.openedition.org/gradhiva/3447

DOI : $10.4000 /$ gradhiva.3447

ISSN : 1760-849X

Éditeur

Musée du quai Branly Jacques Chirac

\section{Édition imprimée}

Date de publication : 6 décembre 2017

Pagination : 100-133

ISBN : 978-2-35744-096-8

ISSN : 0764-8928

\section{Référence électronique}

Katerina Kerestetzi, «Architectures divines. Espace et kinesthésie dans le palo monte afro-cubain », Gradhiva [En ligne], 26 | 2017, mis en ligne le 06 décembre 2019, consulté le 03 janvier 2020. URL

http://journals.openedition.org/gradhiva/3447 ; DOI : 10.4000/gradhiva.3447 


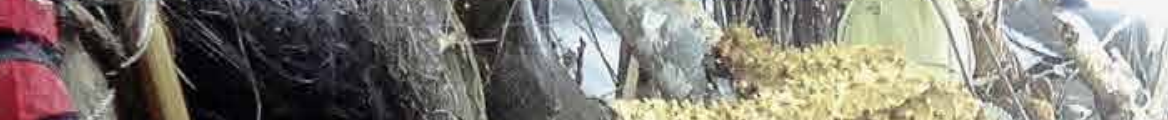
2) $1 x+2$

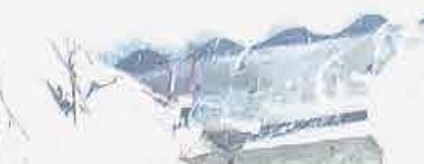

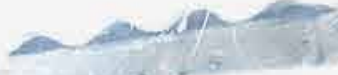

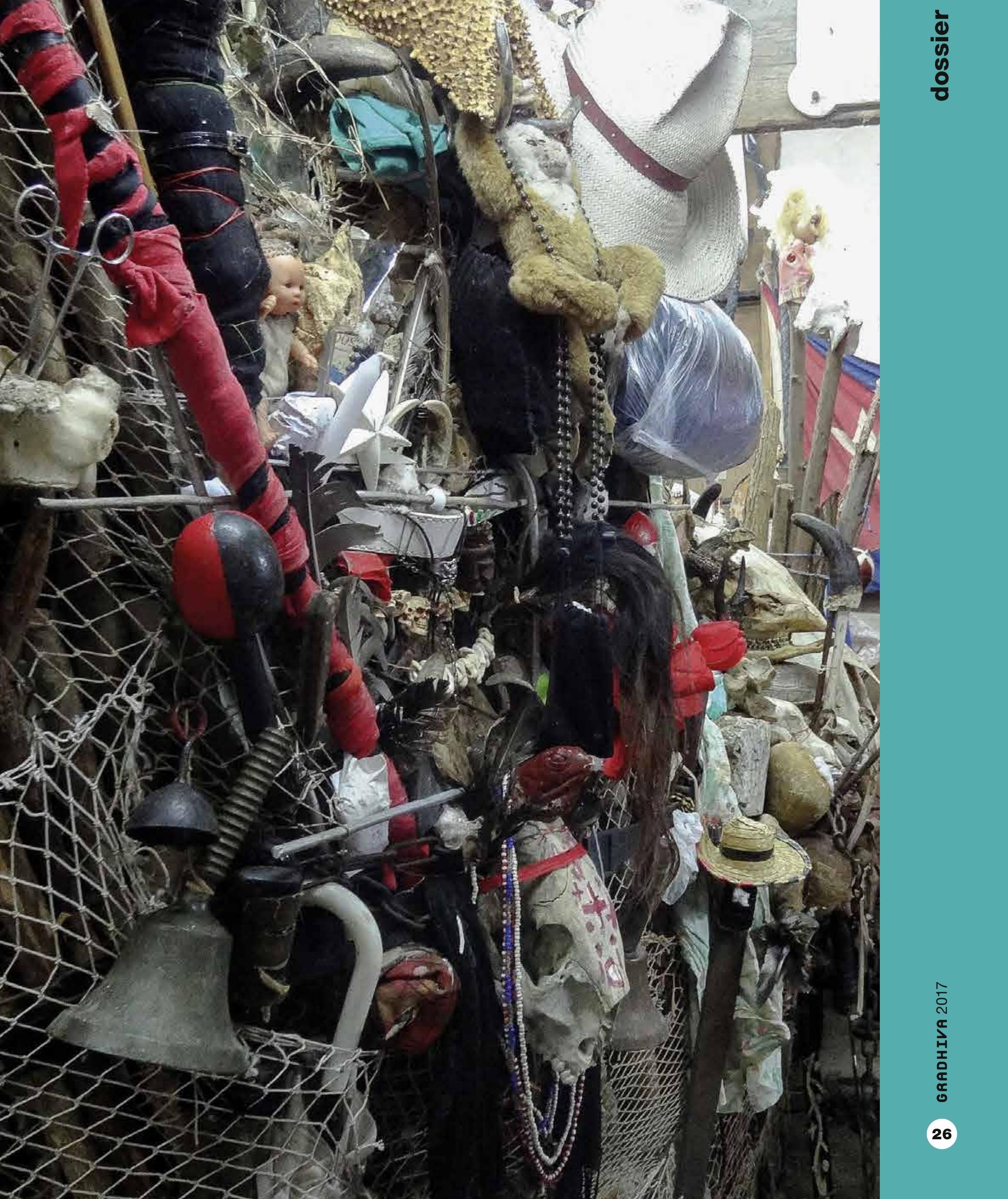




\section{Architectures divines}

Espace et kinesthésie dans le palo monte afro-cubain

par Katerina Kerestetzi

Cet article interroge le rôle des configurations spatiales dans le palo monte afro-cubain. Dans ce culte, chaque chef rituel peut définir sa cosmologie et sa liturgie de manière autonome en créant, entre autres, un espace rituel personnel et personnalisé chez lui, les lieux de culte étant exclusivement domestiques. C'est cette idiosyncrasie architecturale qui nous intéresse ici car elle met en place des schémas moteurs spécifiques qui conditionnent la forme de l'action rituelle. La coordination des mouvements, des attitudes et des postures est induite par la spatialité des sanctuaires, mais aussi par leur acoustique et leur décorum. Chaque lieu de culte peut ainsi devenir source d'innovation rituelle. Comment un élément aussi trivial que la présence de sièges conditionne-t-il la mise en œuvre d'un rituel? Comment la taille d'un artefact détermine-t-elle la mesure de sa sacralité? En comparant le déroulement de l'action rituelle dans différents sanctuaires, à partir de l'étude des affordances architecturales, nous analysons la contingence topologique de cette pratique, autrement dit la dimension spatiale de l'expérience religieuse. 
1. À Cuba, on assiste au foisonnement des églises évangéliques, pentecôtistes, des témoins de Jéhovah, qui se développent au même rythme intense que les religions afro-cubaines. Celles-ci comprennent à la fois le palo monte (Kerestetzi 2016), la santería, le culte d'Ifá, corollaire de la santería d'influence yoruba, culte de divination uniquement masculin; la société secrète masculine des Abakuás (d'origine efik-ibibio) et le espiritismo syncrétique, une variante du spiritisme euro-américain ayant intégré plusieurs éléments liturgiques afro-cubains. La majorité des adeptes pratique simultanément plusieurs de ces cultes. Un Cubain peut à la fois être palero, santero, spirite et catholique. Sur les religions afro-cubaines, voir parmi beaucoup d'autres: Ortiz 1906; Cabrera 2003; Castellanos et

Castellanos 1992; Argyriadis 1999; Palmié 2002.
Le visible et le kinesthésique étant totalement indissociables, la production du sens lors d'un événement visuel ne saurait laisser intact l'état du corps de l'observateur: ce que je vois produit ce que je ressens, et réciproquement mon état corporel travaille à mon insu l'interprétation de ce que je vois. (Godard 1995: 227)

Le palo monte, dont la présence à Cuba procède de l'arrivée des esclaves bantous, fait partie des religions dites afro-cubaines ${ }^{1}$. Ses adeptes, les paleros, concluent des pactes avec certains morts puissants, les nfumbis, dans des cimetières où ils dérobent leurs os pour les rapporter chez eux et les installer, avec quantité d'autres éléments, dans des chaudrons rituels, les ngangas. Ils leur redonnent ainsi une forme de corporéité et de matérialité, nécessaires au développement de leur pouvoir effectif. Les plus dévoués des paleros fondent autour d'eux un groupe d'initiés dont la taille, très variable, peut aller d'une poignée d'individus à plusieurs centaines de personnes. Ces "familles de religion" (familias de religión) sont dirigées par un chef, homme ou femme, considéré comme le «parrain » (padrino) ou la «marraine" (madrina) spirituel(le) de la majorité des membres. II n'existe pas d'autorité religieuse centralisée ou de corpus mythologique suffisamment consistant pour systématiser la pratique de ces groupes. Chaque chef de culte peut ainsi la définir et, pour peu qu'il suive certains protocoles rituels élémentaires, il est libre de construire ses propres cosmologie et liturgie de manière autonome et de se créer un espace rituel personnel.

Cette idiosyncrasie architecturale met en place des conduites et induit des sensations qui conditionnent la forme de l'action rituelle. Nous interrogerons en premier lieu le sens kinesthésique, soit la manière dont la coordination des mouvements (leur orientation, leur direction), des attitudes et des gestes, est induite par la spatialité des lieux de culte et leur topologie, mais aussi par leur acoustique, leur décorum, leurs odeurs et leur luminosité.

Henri Lefebvre (1974) défend l'idée selon laquelle l'espace peut être considéré comme un «analyseur de la société", révélateur des rapports sociaux (de production, de consommation, de domination...). Il s'agit d'appliquer ce constat sociologique à une échelle ethnographique et pragmatique pour mettre en lumière la relation organique entre l'agencement des espaces du culte, les processus cognitifs et les comportements qu'il induit. Par espace, nous entendons l'espace architectural et topographique (ses dimensions, son esthétique, sa configuration, la disposition des meubles et des objets), mais aussi l'espace sensoriel, olfactif et sonore. Nous verrons comment chaque espace du palo monte rend possibles certaines actions (Rapoport 1982) et modalités de communication alors qu'il en condamne d'autres, comment il participe à dessiner des styles rituels, comment même il engendre des formes de religiosité singulières. En effet, certaines actions sont «situationnelles» (au sens de Erving Goffman), elles ne se déroulent pas simplement dans un lieu mais procèdent de chaque lieu (Richardson 2003: 77).

Nous nous intéresserons aux affordances praxiques, sensorielles et relationnelles des lieux du palo monte - le terme d'«affordance » désigne la capacité d'un objet à suggérer sa propre utilisation par sa seule constitution matérielle (Gibson 1977); par exemple, une tasse de café nous invite à la tenir par sa anse. Nous verrons comment la configuration des lieux de culte suggère une manière de sentir, de penser, de se tenir et de communiquer. Nous confronterons les cas de différents sanctuaires et paleros. Puisque l'on 
traite ici des processus individualisants, la question de la personnalité sera également abordée; l'espace que chacun conçoit pour sa pratique trahit certains traits de caractère. Nous verrons que ces dispositions personnelles se muent en composantes du décor palero. Notons que ces processus idiosyncrasiques sont fortement structurants: la réputation et l'efficacité de chaque palero dépend de sa capacité à se distinguer. Cette production de la différence repose en grande partie sur sa sensibilité, ses préoccupations esthétiques et sa manière toute personnelle de concevoir et de bâtir, au sens littéral, sa foi.

Dans une même veine praxéologique, Warnier (2009) a montré comment, chez les Grassfields du Cameroun, l'acceptation de la domination du monarque passe par des interactions avec des objets matériels, des conduites sensorimotrices et des techniques du corps qui permettent d'incorporer la soumission. En mettant en exergue les liens entre architecture religieuse et performance, notre but est ici de présenter les modes d'intériorisation des différentes écologies rituelles. Nous examinerons de quelle manière les architectures divines deviennent des «espaces somatisés » (embodied spaces) [Low et Lawrence-Zúñica 2003] et comment les «dimensions spatiales du comportement» (Amerlinck 2001) rituel ainsi que les effets sensoriels et émotifs de la matière deviennent une variable importante dans l'édification des pratiques religieuses.

\section{Le cuarto de fundamento: esthétique et sensorialité}

Tu as beau être quelqu'un de bien. Moi je le suis et les autres aussi ... en temps normal. Et à l'extérieur du cuarto de fundamento. Mais quand j'entre dans le cuarto, là, je cesse d'être Hector et je deviens Tata Ero [nom rituel]. J'invoque le Diable, je parle avec les esprits infernaux, je fais descendre la lune, les étoiles, le soleil... Parce que je suis ici. (Hector, palero)

Le temple idéal du palo monte est la nature, sauvage. Et si ses adeptes n'étaient tenus, pour des raisons plus ou moins pragmatiques, d'officier chez eux, c'est au milieu des arbres ou des montagnes qu'ils le feraient. D'abord parce que les étendues sauvages sont les réservoirs magiques des matières premières de leur pratique et, en particulier, des palos, ces branches d'arbres d'une variété infinie qui donnent son nom au culte des paleros (littéralement palo monte signifie «branche de la forêt », la forêt - el monte - étant ici entendue comme un synonyme de «nature»). Mais aussi parce que les espaces sauvages sont le lieu de résidence des entités avec lesquelles commercent les paleros: les morts (les nfumbis) et les mpungus ${ }^{2}$. Dans le palo, c'est là qu'est l'au-delà: dans les forêts et les rivières, dans les profondeurs de la mer, dans le tonnerre et le vent ou dans les cimetières, sauvages à leur manière. Mais à Cuba, les contraintes judiciaires et les préjugés obligent les paleros à officier chez eux, «à portes fermées», disent-ils. Le palo monte - comme, dans une moindre mesure, la santería, le culte d'lfá et le spiritisme - ne possède pas de temples publics. II est pratiqué exclusivement dans l'espace domestique, dans la pièce dite le cuarto de fundamento (littéralement «pièce de fondation "), ou simplement cuarto et munanso.

Avant tout, le cuarto de fundamento peut être appréhendé comme une tentative de recréation artificielle de l'environnement naturel des esprits.
2. Les mpungus sont des divinités associées à un lieu naturel ou urbain sur lequel elles exercent un contrôle absolu. Leur rôle dans le culte est secondaire par rapport aux nfumbis, les véritables protagonistes du palo. Voir Cabrera 2003;

Castellanos et

Castellanos 1992;

Argyriadis 1999 ;

Palmié 2002 
Vistus

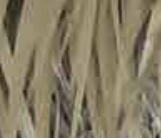

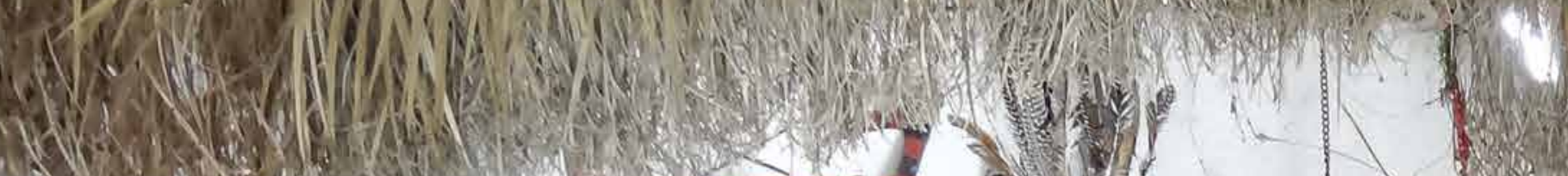

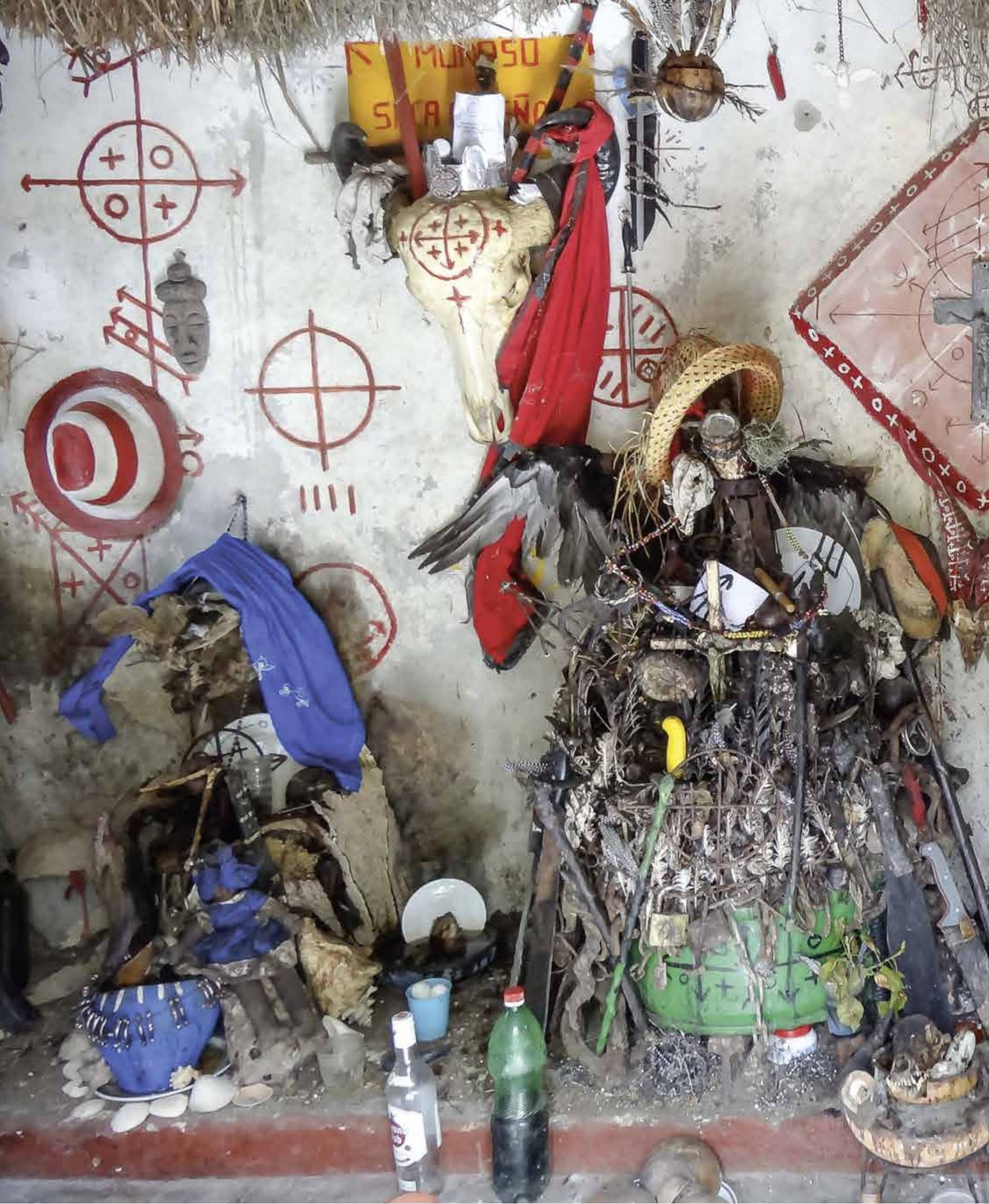




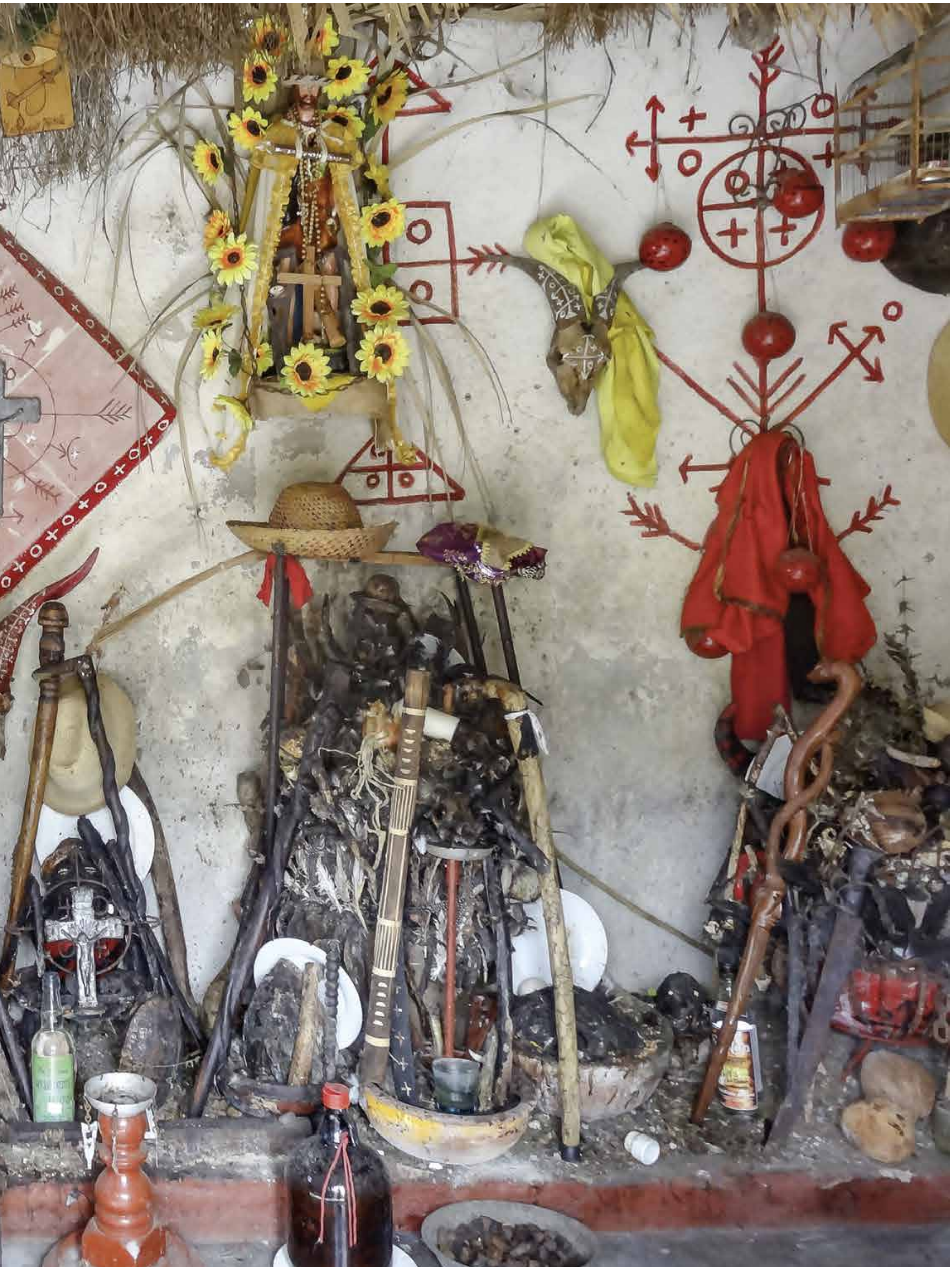


3. Il y plusieurs niveaux d'implication dans le palo monte qui dépendent des contraintes spatiales mais aussi des inclinaisons personnelles; certains peuvent même s'initier au culte presque malgré leur volonté parce qu'un palero le leur a conseillé comme solution plus durable à leurs problèmes.

\section{double page précédente}

fig. 1

Les ngangas.

Palmira, 2015. Photo

Katerina Kerestetzi.
C'est pourquoi il est construit de préférence avec des matériaux issus de substances naturelles, ayant subi peu ou pas de transformation: une cabane en bois, avec un sol en terre battue ou en béton grossier. Le must étant de l'adosser au pied d'une ceiba (le fromager, arbre sacré des paleros). Tout ceci implique évidemment que le palero dispose d'un espace extérieur pour installer sa cabane. Si le cuarto est situé loin de l'espace habité, c'est aussi pour protéger les habitants de la maisonnée de la dangerosité des nfumbis. Car ces esprits jaloux, pour jouir de l'attention exclusive de leur palero, peuvent se montrer cruels avec ses proches, en les frappant de diverses maladies ou en œuvrant contre la paix de leur ménage. Bref, si le pratiquant ne possède pas d'espace extérieur, s'il habite dans un appartement, il va devoir trouver une autre manière d'isoler ses autels. Au mieux, il les enfermera dans une pièce à leur usage exclusif, au pire, il les mettra dans un placard ou sous un lit. Mais il devra alors renoncer à ses ambitions de devenir un palero expérimenté car l'impossibilité de se construire un cuarto est un frein à la pratique rigoureuse du culte et à l'approfondissement de la connaissance qui émerge des échanges avec les ngangas ${ }^{3}$.

Dans un cuarto, on est, en premier lieu, saisi par la quantité et le caractère hétéroclite des objets qu'il contient. La plupart sont posés à même le sol. Généralement, ils sont tous concentrés le long d'une des parois du cuarto, l'ensemble formant une sorte d'autel. Au centre de celui-ci, trône le chaudron de terre ou de fer des paleros: la nganga. Celle-ci est elle-même une expression du foisonnement ambiant puisqu'elle déborde littéralement de matériaux en tout genre: vingt et une branches d'arbre, une chaîne, des plumes, des restes d'animaux, des clous, des crochets... La liste est longue et l'œil se perd à vouloir en identifier tous les éléments. De fait, la nganga est conçue comme un microcosme (Cabrera 2003). Parfois elle est accompagnée de ngangas secondaires ainsi que d'autres objets, plus petits, incarnant des mpungus. Tous sont recouverts de matières organiques en décomposition. Sur le pourtour de la pièce sont entreposées des branches d'arbre, des herbes et des récipients de substances diverses. Le reste de l'espace est vide à l'exception de quelques chaises ou tabourets.

Dans un cuarto, les artefacts ne sont ni sophistiqués ni luxueux, contrairement à certains objets de la santería par exemple. Ils sont faits de matériaux rudimentaires (ciment, bois, noix de coco), grossièrement travaillés et taillés, sans préoccupation esthétique. Même lorsqu'ils figurent une divinité, les traits en sont rarement gracieux ou symétriques. L'ensemble contribue à créer un univers inquiétant. Dans le palo, plus que dans les autres cultes afrocubains, ce sont les pratiquants eux-mêmes qui fabriquent leurs objets. Seuls quelques-uns sont achetés en boutique, ceux qui exigent une technicité que les paleros n'ont pas forcément: c'est le cas des chaudrons et des pots de fer ou terre cuite. Les seules pièces ouvragées que l'on peut trouver dans un temple du palo monte sont des objets de ferronnerie, tels que les machettes, menottes, chaînes, vieux pistolets ou clous. Ceux-ci sont utilisés pour leur puissance mais aussi peut-être pour leur aspect rude et naturel donnant une impression d'authenticité. Selon Juhani Pallasmaa (2005: 31), «les matériaux naturels - la pierre, la brique, le bois - permettent à notre vision de pénétrer leur surfaces et nous permettent d'être convaincus de la véracité de la matière. Les matériaux naturels expriment leur âge et leur histoire, ainsi que l'histoire de leurs origines et l'histoire de leur usage humain ". 
L'environnement matériel palero procure une certaine fascination qui trouve sa source dans la peur. Après tout, il s'agit bien d'évoquer la présence de morts oscuros ("obscurs»), d'où l'esthétique morbide du cuarto - les chairs en décomposition peuvent ainsi être envisagées comme un principe décoratif - et agressive - avec toutes les armes de fer sur l'autel. La saleté et le désordre des pièces - parsemées de déchets rituels et des matières premières destinées aux opérations magiques: branches, feuilles, ongles, cire, cheveux, cigares écrasés, photos, tâches de sang - sont la toile de fond de l'univers palero. L'inquiétude que les morts peuvent provoquer chez ceux qui pénètrent dans le cuarto est ainsi dédoublée par le sentiment d'être pollué, souillé, au sens de Mary Douglas (1981) pour qui le désordre et la saleté, dépourvus de structure, sont associés à l'immoralité et au danger. On voit ici comment l'expérience sensorielle devient représentation théologique.

Le sens visuel n'est pas le seul à être affecté par cette étrangeté perturbante. Tout dans un cuarto palero contribue à susciter le frémissement religieux. L'odeur nauséabonde du sang en putréfaction qui émane des objets abreuvés, combinée à celle de la fumée de tabac et du rhum des libations, aigrissent l'ambiance olfactive et participent au basculement immédiat dans l'univers du culte, évoquant à la fois les forces vitales qui animent le temple et la mort qui lui confère sa toute-puissance. Comme le remarque Arnaud Halloy (à paraître) pour le cas du xângo au Brésil dont l'univers sensoriel ressemble à celui des paleros, l'odeur du sang constitue un «attracteur perceptuel » qui provoque de fortes émotions. Selon lui, les odeurs d'un rituel sont constitutives de son efficacité et leur apprentissage involontaire au fur et à mesure de la participation est susceptible d'induire des émotions religieuses singulières qui seront ensuite "attachées" à ces odeurs et enclenchées automatiquement par elles. Le cuarto palero a une identité olfactive très caractéristique que chacun associera à son expérience personnelle du culte.

L'obscurité de la pièce intensifie également les sensations déstabilisantes. En effet, l'utilisation de l'éclairage électrique contrevient aux principes du palo. Au même titre que les autres composantes de ses autels, la lumière doit être naturelle; la seule lumière artificielle que l'on accepte est celle produite par la flamme de la bougie, allumée en permanence au pied de la nganga. Quant à la lumière naturelle, elle n'abonde pas non plus, puisqu'on officie traditionnellement portes et fenêtres fermées pour soustraire aux regards indiscrets les secrets des rituels. Pour Pallasmaa (2005: 47), «la lumière claire homogène paralyse l'imagination de la même façon que l'homogénéisation de l'espace affaiblit l'expérience de l'être et efface le sens du lieu. La brume et la pénombre réveillent l'imagination, en rendant les images visuelles floues et ambiguës ». Le ballet des ombres chorégraphié par les oscillations de la flamme des bougies et la lumière qui filtre par les interstices des parois du cuarto animent les formes et les rendent encore plus énigmatiques. La cabane de bois du palero réussit ainsi haut la main le défi de distiller et d'instiller chez ses visiteurs le mystère espéré.

\section{Spatialité et personnalité: l'idiosyncrasie de l'espace}

La singularité esthétique des cuartos paleros tient beaucoup aux goûts, ambitions et talents de chaque pratiquant. En confrontant les cas de deux d'entre eux, formés auprès du même initiateur, nous interrogeons ici 


\section{ci-contre}

fig. 2

Le cuarto de Pedro Tomás. Cienfuegos, 2004. Photo

Katerina Kerestetzi. la part de personnalité qui y est «disséminée» (Gell 1998: 26). Les liens entre la personnalité des paleros et leur espace cérémoniel rendent en effet l'expérience rituelle plus intelligible en quelque sorte, créant un «terrain d'entente » (Hanks 2009) entre les différents acteurs de l'interaction rituelle, indépendamment de leurs connaissances du palo.

Une petite cabane en bois, aux contours irréguliers, installée au fond d'une cour, entre un poulailler et un clapier: nous sommes devant le cuarto de Pedro Tomás qui, comme les autres paleros cités, habite Cienfuegos, la ville où nous avons conduit l'essentiel de notre recherche. Une porte branlante donne accès à un espace de quatre mètres sur deux. L'endroit n'a pas de fenêtres, il est plongé dans l'obscurité. Pas de doute, il respecte les principes du cuarto de fundamento idéal.

Ce cuarto est modeste et austère: son volume est petit, sa décoration rudimentaire. II ne comporte qu'une seule nganga, de taille réduite. Les artefacts rituels qu'on y trouve sont minimalistes, d'une facture amatrice et sans sophistication (fig. 2). II semblerait que Pedro Tomás ait délibérément choisi de négliger l'aspect esthétique de son espace de culte. Pourtant l'esthétique compte parmi ses préoccupations, on pourrait même dire qu'il est coquet. Son cuarto, en revanche, n'est pas conçu pour impressionner: c'est un lieu de travail, car comme c'est souvent le cas, Pedro Tomás a fait du palo son métier. Deux chaises sont posées de part et d'autre de l'autel - une pour lui, l'autre pour son client. L'ensemble forme l'espace de consultation.

D'autres indices trahissent le caractère fonctionnel du cuarto, à commencer par le grand nombre d'artefacts dédiés aux opérations magiques ou trabajos, comme les tratados, ces petits paquets contenant des poudres, des feuilles pulvérisées, des photos, des ongles et des cheveux des clients et de leurs victimes. Même les choses qui semblent n'avoir aucune utilité peuvent en cacher une, comme cet iguane desséché qui trône en bonne place sur l'autel et qui n'est autre qu'un réceptacle rempli de substances magiques, destiné à anéantir les ennemis du palero. Voilà un exemple typique d'élément magique opportuniste: le palero, qui n'avait sans doute jamais songé à travailler avec un iguane, a eu par hasard la chance d'entrer en possession de ce reptile et a simplement saisi l'occasion pour l'intégrer à sa pratique. Après sa décomposition, c'est une chouette, la nsunsundamba qui a pris sa place. La visibilité de ce type d'«outillage» contraste avec la modestie des artefacts qui représentent les divinités. Lucero, par exemple, celui qui ouvre et ferme les chemins des hommes, prend la forme d'une tête de ciment haute d'une vingtaine de centimètres. Ses traits sont grossièrement modelés, trois cauris figurant ses yeux et son nez.

La primauté des artefacts strictement fonctionnels de Pedro Tomás et le minimalisme de son cuarto indiquent les motivations de sa pratique religieuse et, par extension, reflètent certains traits de sa personnalité, notamment son pragmatisme. Pedro Tomás est quelqu'un qui cherche avant tout, par sa pratique, à améliorer ses conditions de vie. Il se sert de sa nganga pour quitter le pays, pour s'enrichir... Ce n'est pas un besoin de reconnaissance qui l'a poussé à fonder un groupe, contrairement, semble-t-il, à son confrère Elier.

D'une surface de quinze mètres carrés le cuarto d'Elier est presque deux fois plus grand que celui de Pedro Tomás. Contrairement à ce dernier, le palero n'a pas ménagé ses efforts pour en soigner l'aspect. Plus «moderne", il a remplacé les parois de bois par des murs de ciment et engagé un peintre pour composer une impressionnante fresque colorée, 


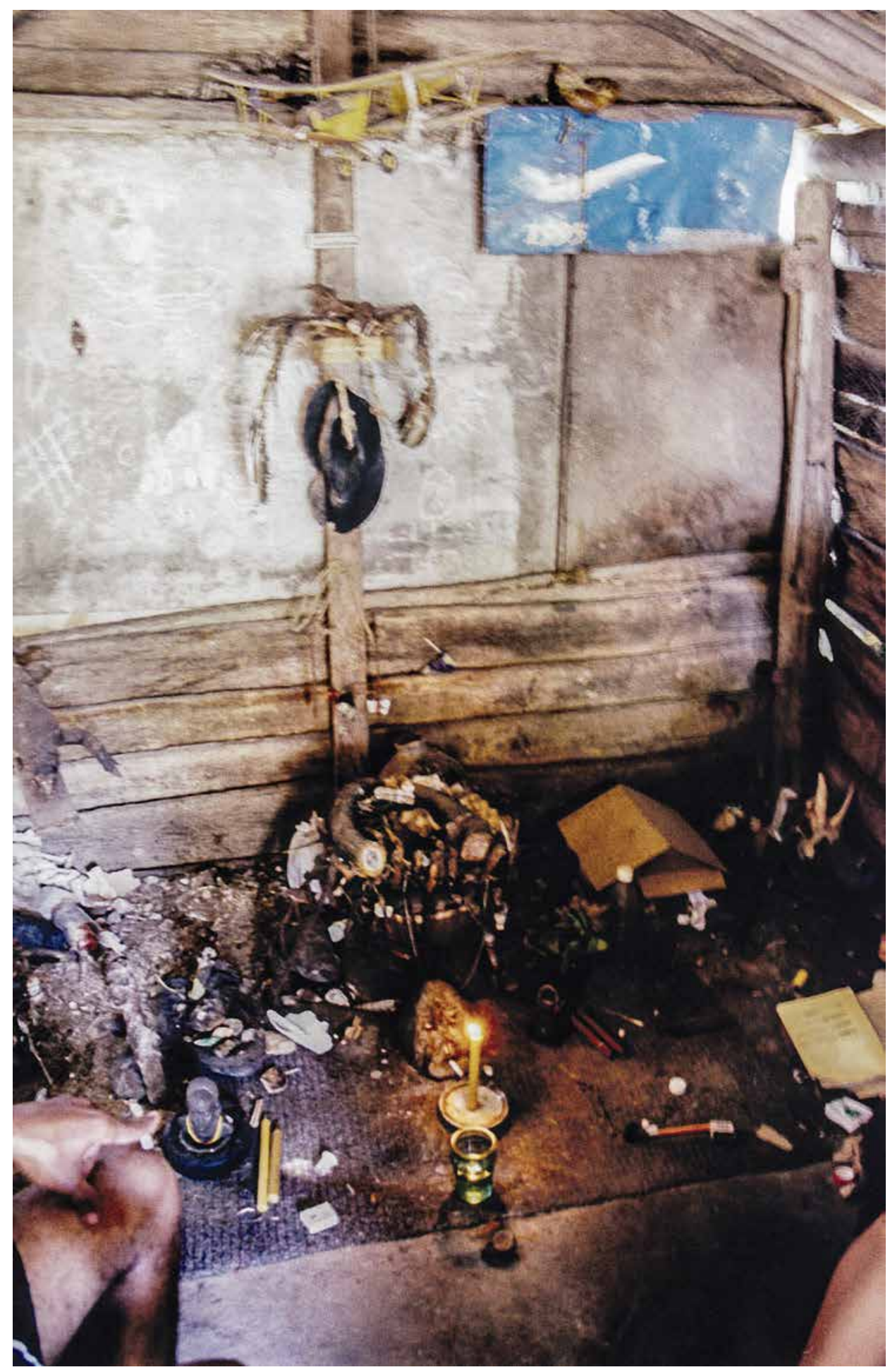



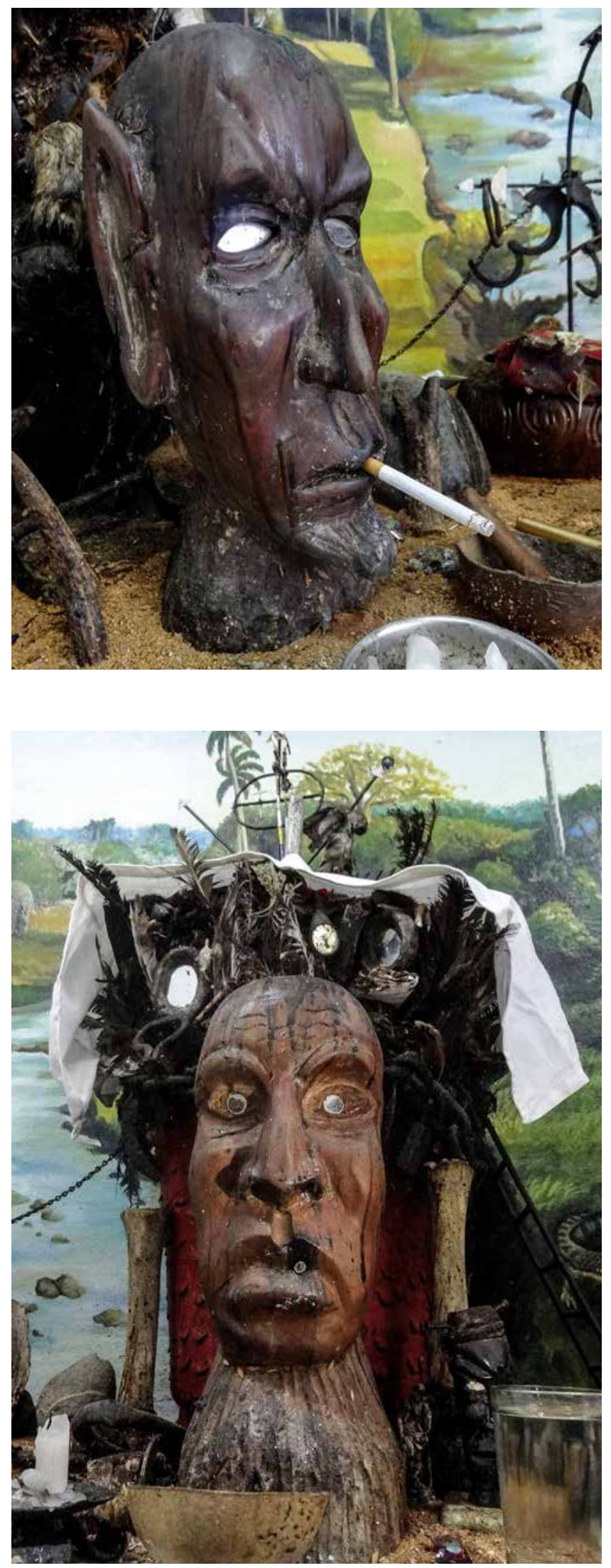
avec des paysages de forêts tropicales, des crocodiles et des éléphants ainsi que d'autres cuartos de fundamento - en l'occurrence celui de son initiateur, représenté par la cabane à droite (fig. 5) -; bref, l'exigence de recréation naturelle a pris chez lui une forme picturale.

Tout dans son cuarto est pensé dans l'intention d'impressionner ses visiteurs, parfois au prix de dépenses non négligeables compte tenu des faibles revenus des Cubains. L'autel a fait l'objet d'un traitement particulier: en occupant le mur le plus large, il absorbe le regard avec ses deux imposants chaudrons peints, aux motifs assez raffinés. Devant chaque chaudron est disposée une statuette de bois à l'effigie de la divinité Lucero dont les traits sont finement ciselés, consciencieusement polis et vernis. Elles sont l'œuvre d'un sculpteur sur bois professionnel, initié par Elier (fig. 3 et 4). À cette préoccupation esthétique, s'ajoute une volonté d'ordre, de propreté et d'organisation inhabituelles pour un cuarto. Tout est en effet bien rangé chez Elier; il a même installé un placard à «pharmacie» magique. Comparé à l'aspect désordonné du cuarto de Pedro Tomás, qui trahit une certaine insouciance, celui-ci respire l'anxiété et la volonté de contrôle. En effet, ce palero est très soucieux de son image, d'où le fait que son cuarto, extension matérielle de lui-même, soit particulièrement bien entretenu.

Si ses proches le consultent parfois, Elier n'est pas un praticien «professionnel» qui reçoit des clients contre rétribution, ce qui explique l'absence de traces d'opérations magiques chez lui. Comme il l'avoue lui-même, sa pratique est motivée par une recherche d'émotion et par un intense besoin de communication avec ses morts. Alors que Pedro Tomás se rend tous les matins dans son cuarto demander conseils et faveurs, lui ne s'y rend que tous les trois jours pour «méditer » et «ramasser de l'énergie». On pourrait dire que l'un y cherche à mieux agir et l'autre à mieux sentir. Alors que pour Pedro Tomás ces visites sont des moments plaisants - il y boit du rhum, fume des cigarettes, se socialise -, Elier, lui, les accomplit avec sérieux et intensité:

Quand je viens ici la nuit, tout est éclairé. J'ai mis des étoiles autocollantes phosphorescentes et, en plus, j'allume mes bougies. C'est très beau. La nuit je viens ici faire mes «choses». Et je me transforme, je me peins en diable, je danse nu... Et quand je ne me sens pas bien, je viens dormir ici. Quand je me réveille, parfait, je ne ressens plus aucune douleur. Je ne sais pas si c'est psychologique. Je ne peux pas l'expliquer.

Cette ritualité pour le moins inhabituelle a pourtant permis à Elier d'accoucher d'un lieu de culte à sa mesure. Cette expression spatiale de dispositions personnelles est, en soi, une forme de communication, un «interactionnisme symbolique» (Rapoport 1982: 59) qui se joue au niveau sensoriel et qui structure l'expérience de la consultation. L'aspect fonctionnel du cuarto de Pedro Tomás peut amener par exemple un client à pressentir son efficacité et à faire confiance à son travail. La modestie et la simplicité de l'agencement parviennent, malgré toutes les présences inquiétantes, à ne pas l'intimider et à donner un ton décontracté et même familier à sa consultation. Dans le cas d'Elier, l'aspect plus luxueux de son cuarto, l'absence d'un espace prévu

\section{ci-contre}

fig. 3 et 4

Les Luceros d'Elier.

Cienfuegos, 2015. Photo

Katerina Kerestetzi. 


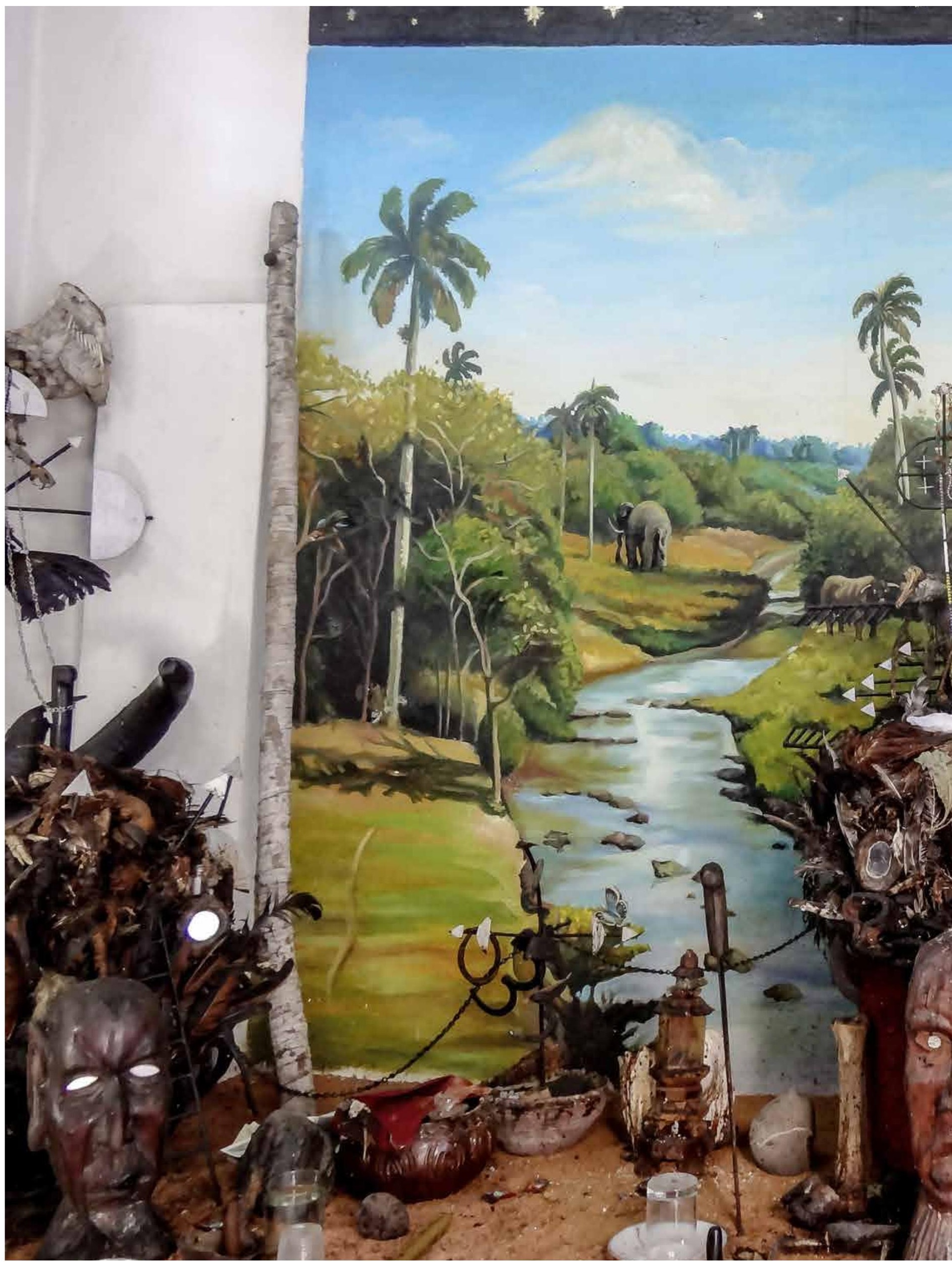


double page précédente

\section{fig. 5}

Le cuarto d'Elier. Cienfuegos, 2015.

Photo Katerina Kerestetzi.

\section{ci-contre}

fig. 6

Nganga syncrétisée avec Ogún, divinité santera des métaux. Cienfugos, 2015.

Photo Katerina Kerestetzi. pour le visiteur, sa propreté et son ordre, trahissant une certaine «inutilisation ", peuvent suggérer qu'il s'agit d'un espace conçu non pas pour le travail magique (artisanal, désordonné) mais plutôt à des fins liturgiques ou méditatives.

\section{Espace somatisé: le sens kinésique}

Comment la topologie d'un cuarto influence-t-elle l'action rituelle? Les écarts de morphologie, de taille, la mise en scène des objets, prédisposent aussi bien à des formes d'action singulières qu'à la mise en place de schèmes moteurs spécifiques. Notre mouvement dans l'espace et notre positionnement par rapport aux autres (et aux objets), c'est-à-dire notre comportement "proxémique" (Hall 1974), sont fortement influencés par nos représentations et des schèmes moteurs préétablis dans le cerveau qui dépendent en partie de nos expériences passées (Berthoz 1997). Par exemple, celui qui est au courant du danger que représente une nganga se garde, consciemment ou pas, à bonne distance de celle-ci. Pedro Tomás et Elier ont agencé leur cuarto de façon diamétralement différente, on l'a vu. L'enjeu est maintenant de montrer comment cette différence topologique influe sur les composantes des rituels. Nous verrons s'opposer un «nganga-centrisme " propre au cuarto de Pedro Tomás et un «ego-centrisme » chez Elier.

Posée au centre exact de l'autel, faisant bien face à la porte d'entrée, la nganga de Pedro Tomás est l'élément que l'on perçoit en premier en pénétrant dans la pièce. II s'agit par ailleurs de l'artefact le plus élaboré, placé à bonne distance de tous les autres objets. Toutes les lignes de fuite de l'espace convergent vers ce chaudron: la présence de deux rangées de tabourets placées le long des murs du cuarto accentue encore cet effet, le regard étant à coup sûr dirigé vers celui-ci. L'usage des tabourets est quasiment systématique dans ce type de cuarto rectangulaire où les ngangas sont posées à même le sol: les pratiquants, assis bas au niveau du chaudron, respectent de cette façon le principe de la communication frontale; la nganga étant considérée comme une entité vivante et personnifiée à qui l'on s'adresse de face.

L'une des conséquences performatives de cet agencement ngangacentrique est une activité rituelle principalement centripète: la structure interactionnelle des rites organisés par Pedro Tomás est en effet principalement tournée vers le chaudron. Ceci est particulièrement remarquable au début des cérémonies, lorsqu'il dialogue avec sa nganga. À l'aide d'une méthode divinatoire, il interroge de manière répétée le chaudron sur les actions à accomplir pour progresser dans le rituel. La nganga peut par exemple lui indiquer le nom de la divinité qu'il faut mobiliser ce jour-là ou la nécessité de nettoyer rituellement le cuarto. Pour les participants, c'est donc la nganga qui prescrit les actes qui vont jalonner la cérémonie. Cette manière de procéder confère du dynamisme au rituel: en l'absence d'un protocole immuable, on serait tenté de dire que chez Pedro Tomás, les rituels s'auto-organisent.

Pendant la première moitié du rituel, les autres participants restent relativement passifs. La présence d'emplacements fixes contraint doublement leur mouvement en les invitant à la fois à rester assis et à maintenir le regard sur le couple constitué de la nganga et du palero en chef. Tout change dans la seconde partie du rituel, lorsque l'esprit du chaudron vient posséder un médium. L'attention des pratiquants se détache alors de l'autel pour venir se fixer sur lui. À ce moment-là, le médium devient, pour ainsi dire, responsable 


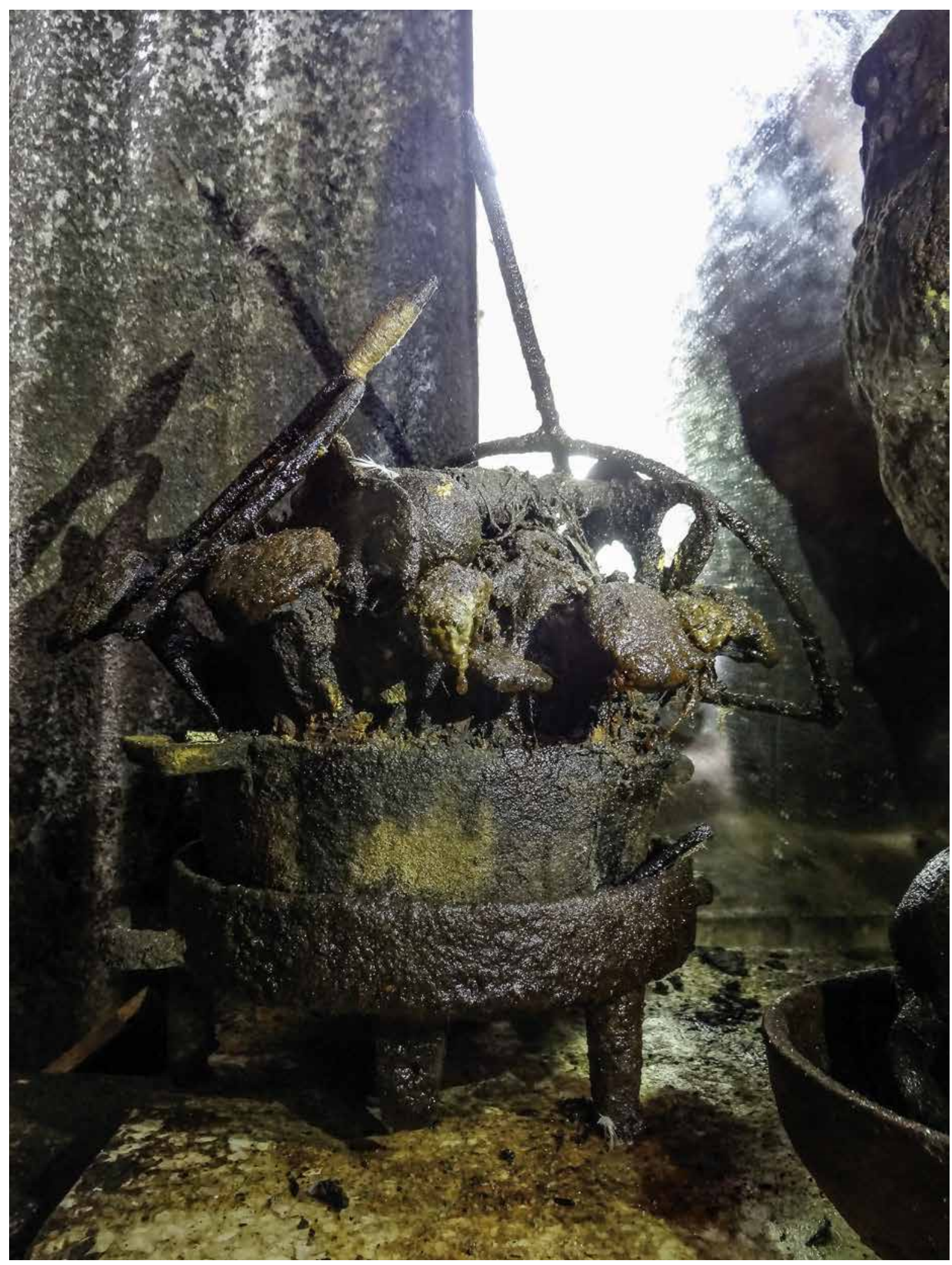




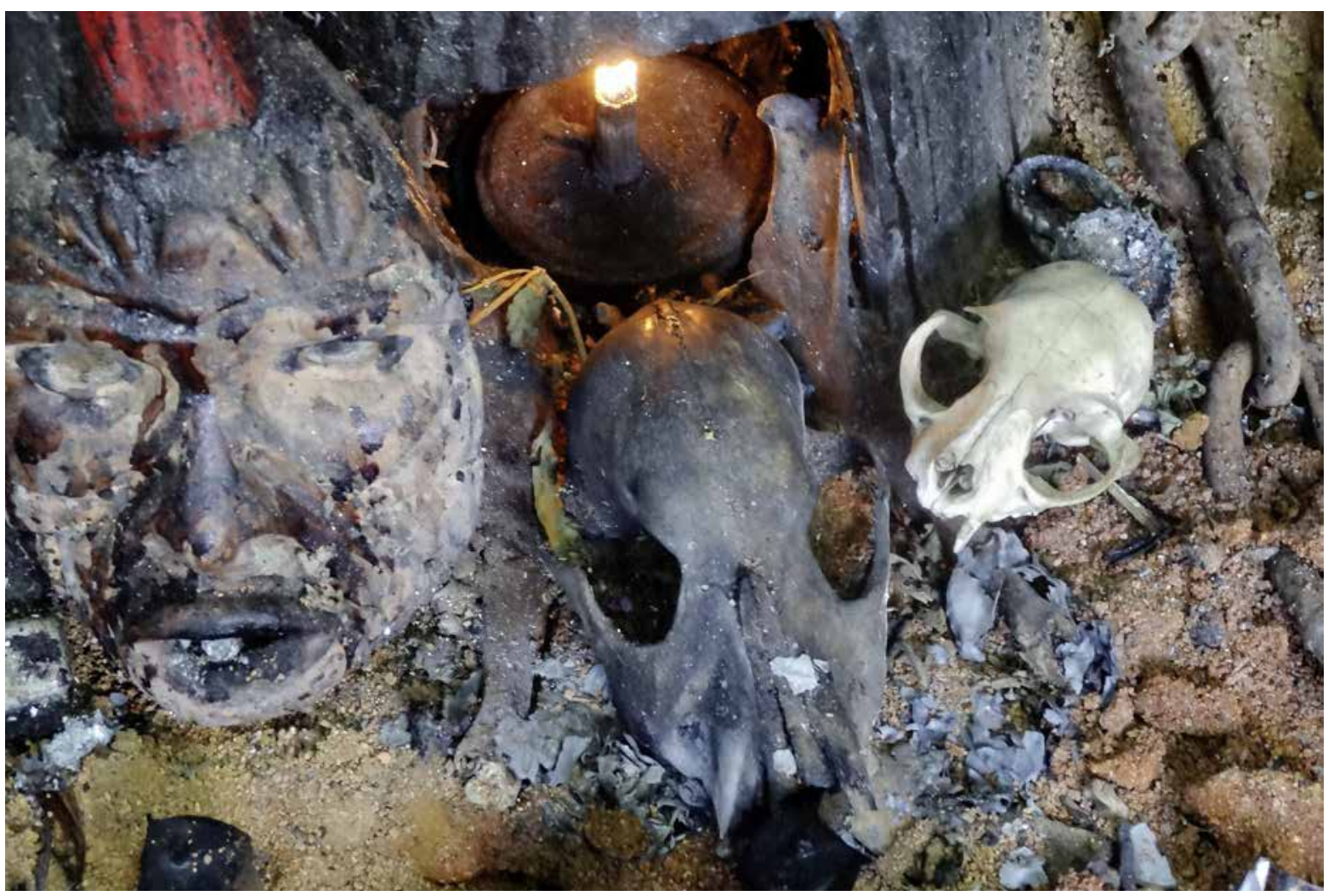

fig. 7

Détail d'un cuarto de

fundamento. Ces esprits

auxiliaires assurent la

protection d'une nganga.

Cienfuegos, 2015. Photo

Katerina Kerestetzi. 
de l'organisation du rituel. Sa performance, ses prophéties, ses danses violentes, en rythment le déroulement. Au cours de cette phase, les participants se lèvent de leur siège pour suivre les déplacements du médium et communiquer avec lui. Pour reprendre l'expression de Nancy Munn (1996), ses mouvements créent «un champ spatial mobile» qui développe la mobilité des participants tandis que ses déplacements génèrent à chaque fois de nouveaux centres interactifs.

Le cuarto d'Elier se différencie de celui de Pedro Tomás tout d'abord par son volume - il est plus vaste - et par sa configuration car il s'étale tout en longueur. La partie réservée aux initiés est vide, sans chaises ni tabourets. Ceux-ci restent donc debout, formant un demi-cercle autour de la nganga centrale. Les hommes sont positionnés d'un côté et les femmes de l'autre. Compte tenu du peu de recul dont ils disposent, l'autel ne peut être saisi tout entier d'un seul regard. Les visiteurs n'en ont qu'une vue fragmentée, condamnés à le balayer des yeux et à porter alternativement leur attention sur l'une ou l'autre de ses composantes. Par ailleurs, parmi les objets rituels, tous plus élaborés les uns que les autres, aucun en particulier ne se démarque par sa taille ou son raffinement. Enfin, la posture debout exclut les ngangas, posées à même le sol, du champ de vision des participants: il faut se pencher pour les voir. Cette configuration spatiale n'offre donc aucun foyer spontané de l'attention. Le seul élément qui s'impose véritablement à la vue est le chef rituel lui-même. Elier est positionné debout, devant sa nganga, de sorte qu'il en dissimule une partie à certains. Tous les regards convergent vers lui (alors que chez Pedro Tomás, seule la nganga est visible de tous en permanence). Cela n'est sans doute pas un hasard. Elier ne conçoit pas le rituel comme une fête religieuse mais plutôt comme un terrain propice à la mise en scène de son autorité. Reposant presque exclusivement sur sa seule performance, ses rituels sont de nature plus unilatérale.

\section{Un espace ouvert: d'une sensation l'autre}

Héctor, lui aussi chef de culte, officie dans un cuarto rectangulaire d'environ deux mètres sur six, situé dans la cour intérieure de sa maison. Mais à la différence d'autres paleros, Héctor n'a pas bâti de cuarto. Sa cour l'est devenue le jour où il y a installé sa nganga. Celle-ci occupe l'une des extrémités de l'espace, protégée par une simple plaque de tôle fixée sommairement. Les cérémonies se déroulent donc en plein air, bien souvent sous un ciel étoilé, "quand les morts sortent ». Mais cette ouverture aux éléments climatiques a une incidence sur l'expérience sensorielle du lieu et du rituel. Alors que les pièces fermées concentrent les odeurs, ici elles se dissipent, suivant l'intensité du vent et l'humidité de l'air. Depuis l'autre bout de la cour, loin de l'autel, seules parviennent celles des cigares et des effluves de transpiration. Ce sont là plutôt des odeurs banales qui ne rompent pas avec l'expérience olfactive ordinaire. Le pouvoir évocateur de l'odeur du sang frais des sacrifices qui suinte sur les ngangas, des carcasses en décomposition, ou de celle du rhum que l'on crache sur l'autel s'est ici évaporé.

Le son s'y perd aussi, du fait de la longueur de la cour qui garde certains participants à distance. Dans cette configuration, on doit élever le ton de sa voix pour s'adresser à la nganga et aux autres initiés. Ainsi Héctor chante assez fort, accompagné par les percussions des musiciens qu'il engage à chaque rituel (contrairement à d'autres qui se chargent eux-mêmes 
Geraldo et sa nganga.

Cienfuegos, 2012.

Photo Katerina Kerestetzi. de l'animation musicale de leurs rites), comme si l'intensité du son venait ici suppléer les odeurs évanouies à ciel ouvert. Des sons assourdissants des percussions, on passe sans transition à des moments de silence qui, par contraste, font vibrer les oreilles et sentir son propre pouls.

Héctor est lui-même danseur et chanteur professionnel. Son goût pour la performance artistique trouve un terrain fertile dans l'agencement de son cuarto. La nganga est posée sur un trépied si haut qu'elle atteint une taille humaine. Là, on est obligé de se tenir debout pour interagir avec l'objet. La posture debout confère de l'amplitude au mouvement; elle donne la possibilité de s'éloigner physiquement de l'objet et de déplacer l'action rituelle ailleurs dans l'espace. Cette situation rend les rituels interactifs et, de fait, plusieurs centres de l'action se mettent en place: l'un, rythmique, autour des musiciens, un autre, "sacré", autour de l'autel, et un troisième, actif, au centre de la cour, laissé vide pour les purifications, les divinations et autres opérations.

\section{La spatialité de l'innovation: la dimension de la croyance}

Geraldo a édifié une nganga gigantesque. Une fabrication régie par les principes de multiplication, de grossissement et de prolifération. Pour lui, la compacité et l'opacité de la nganga ne suffisaient pas, il en a donc fait éclater les limites. Difficile de résister à l'hypothèse qu'il aurait cherché à reproduire dans sa nganga la massivité de son propre corps (fig. 8). Gigantesque comme lui, elle illustre l'idée d'une isomorphie entre sa corpulence et l'architecture de son sanctuaire. Selon James Fernandez (2003: 200, traduction de l'auteur), «les idées que les êtres humains se font d'un lieu sont toujours pour partie une projection de l'image de leur propre corps et, vice versa, leur propre image corporelle est une introjection de leur expérience dans ces lieux [...]. C'est à partir d'eux-mêmes que les hommes se figurent l'espace et en tirent des propriétés qu'à leur tour ils y projettent ».

Les adeptes sont ici conduits à adopter dans leurs interactions avec le chaudron une gestuelle distincte de celle induite par une nganga aux dimensions moindres. Au principe dialogique habituel de la communication frontale entre le palero et sa nganga se substitue ici une autre modalité, plus diffuse, qui voit l'adepte se tourner sélectivement vers les éléments de son environnement les plus adaptés à son intention rituelle. L'impossibilité du face-à-face est ici compensée par une méthode d'invocation empruntée à la santería, qui consiste à appeler une divinité en faisant sonner la cloche qui lui est associée; chaque oricha répond au timbre d'une cloche particulière, qui se distingue des autres notamment par sa forme. Geraldo possède plusieurs clochettes qu'il a posées devant chaque agent spirituel important de son sanctuaire afin de pouvoir facilement le convoquer quand il en éprouve le besoin.

Mais les conséquences performatives de ce gigantisme ne s'arrêtent pas là. La forme de l'objet n'est pas propice au ressenti de l'intimité que les paleros éprouvent face aux ngangas. L'identification de cet objet avec le mort est court-circuitée car il est difficile de l'envisager sous un aspect anthropomorphe. Et où poser le regard dans cette masse impressionnante de matières indiscernables? Cette nganga immense s'en trouve cantonnée à une fonction collective, mobilisée lors des rituels qui rassemblent la communauté et pour les opérations magiques. Pour se recueillir et demander conseil, pour retrouver 


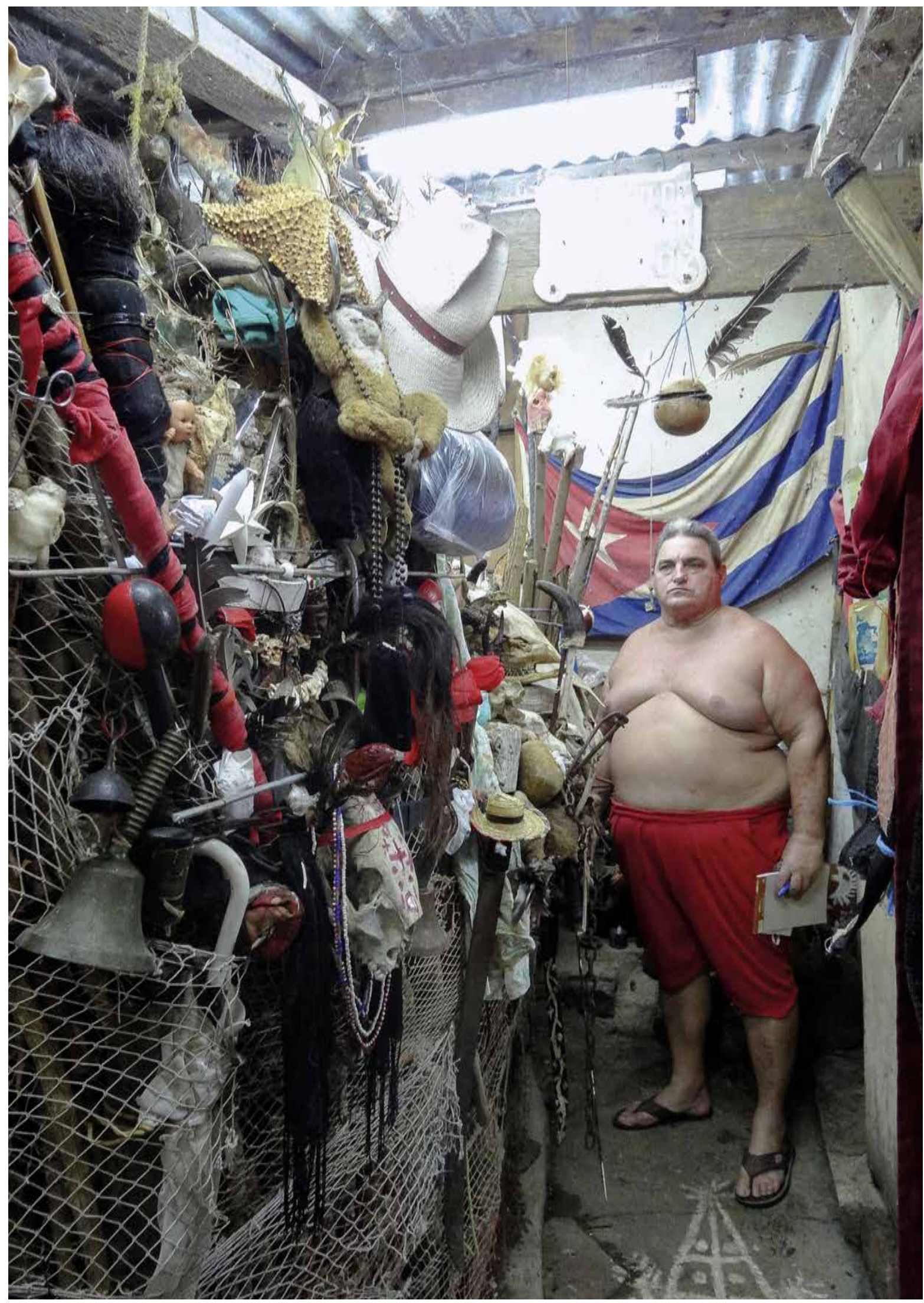




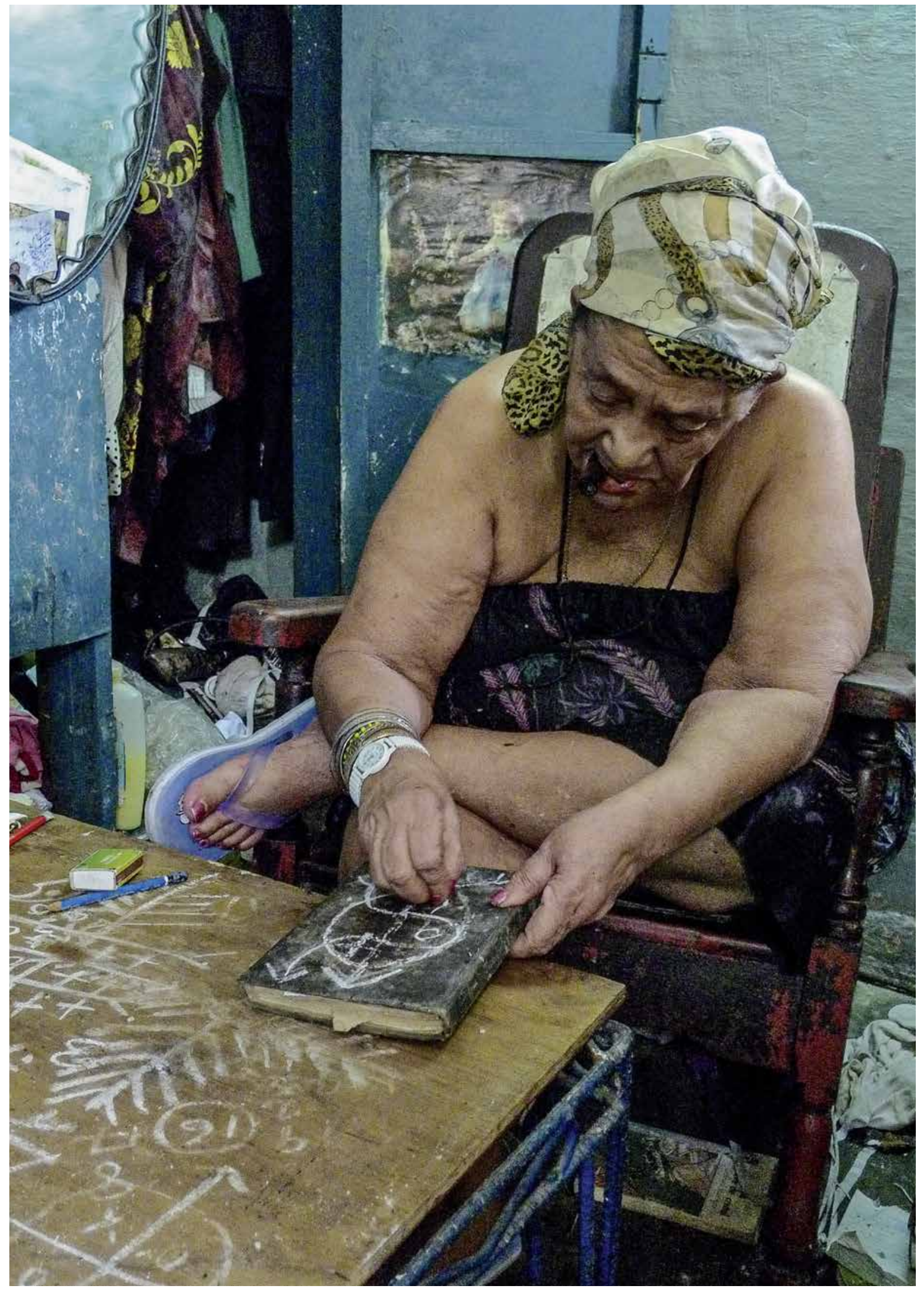


le côté confidentiel des interactions entre les paleros et leurs morts incarnés, Geraldo a bâti une autre petite pièce dans son sanctuaire où il a installé une deuxième nganga, aux dimensions plus classiques. C'est une illustration éloquente de la manière dont les affordances de l'espace projettent des fonctions différentes sur les objets présents. En changeant de forme, la nganga, d'ordinaire polyvalente, a acquis une spécialisation. Cette division des tâches, dédoublée par celle des espaces, est d'autant plus évidente que le palero a donné des noms différents à chacune de ses deux ngangas. La petite s'appelle «nganga» et la grande, «prenda». Pour tous les autres paleros, les deux termes sont parfaitement interchangeables.

Si les contraintes spatiales sont autant de facteurs individualisants de la praxis, elles participent aussi à la définition des hiérarchies dans le groupe. Raúl, l'un des initiés de Geraldo, nous a confié que pour pouvoir devenir initiateur lui-même et acquérir son autonomie religieuse, il lui fallait obligatoirement obtenir le grade de mayombero. Or le seul moyen d'y parvenir est de confectionner une nganga aux dimensions de celle de son initiateur. Le problème est que Raúl vit dans un petit appartement qu'il partage avec sa mère, catholique de surcroît. Pour créer un artefact aussi massif, Raúl n'a donc d'autre solution que de déménager dans une maison individuelle et pourvue d'une cour. Précisons que le grade de mayombero n'existe pas ailleurs dans le palo monte. C'est un échelon hiérarchique créé de toutes pièces par Geraldo - mayombero étant un synonyme exact de palero. Dans les autres maisons de culte du palo monte, le droit d'initier est concédé automatiquement lorsqu'on acquiert une nganga personnelle, peu importe sa taille. Donc, dans le cas de Geraldo, l'espace, plus qu'une ressource, constitue un catalyseur de praxis, de créativité et d'inventivité. Sa matérialité peut même être à la source d'un certain pouvoir sur les initiés: celui qui n'a pas la possibilité de se construire un sanctuaire à l'image de son initiateur est condamné à la dépendance. L'impuissance religieuse est ici proportionnelle à la pauvreté matérielle et la hiérarchie rituelle se mesure à la taille des artefacts.

\section{Spatialité et relations religieuses: épicentres d'actions}

Poursuivons maintenant notre tour d'horizon des impacts de l'organisation spatiale sur les pratiques religieuses en nous intéressant à la relation thérapeutique qui se noue entre le palero et ses clients. L'enjeu est de montrer comment ceux-ci sont affectés par «l'espace-cadre», au sens du frame space de Goffman (1987), c'est-à-dire le cadre physique et virtuel de la consultation. Selon lui, l'espace-cadre n'est pas la simple toile de fond de l'interaction mais en détermine la dynamique. Pour le montrer, nous nous intéresserons au cas de Marelis, âgée de 75 ans, et de son fils, Andrés, un palero d'une cinquantaine d'années. Le choix de comparer une mère et son fils n'est pas anodin. En dépit d'une grande affinité opératoire, l'espace dans lequel chacun d'eux évolue influence de manière déterminante leur efficacité et leur réputation.

À Cienfuegos, Marelis est considérée comme une sorcière puissante et sans scrupules. Sa renommée est telle que, chaque jour, une foule de clients vient se presser devant sa porte dans l'attente d'une consultation. De nombreuses histoires circulent sur son compte. La plus connue raconte comment, dans sa prime jeunesse, elle fut prise en flagrant délit de profanation de tombe alors qu'elle extrayait de terre le mort qu'elle avait choisi pour

\section{ci-contre}

fig. 9

Marelis marque des

firmas sur un livre.

Cienfuegos, 2012.

Photo Katerina Kerestetzi. 
rejoindre sa nganga. Un administrateur du cimetière ayant été témoin de la scène l'avait dénoncée aux autorités - à Cuba, la profanation est passible de trente ans d'emprisonnement. Par chance, cet unique témoin se suicida peu de temps avant le procès et Marelis fut acquittée. Les paleros de Cienfuegos racontent souvent cette histoire avec admiration; pour eux, c'est le mort qui a poussé au suicide l'administrateur afin de protéger sa palera bien-aimée. Ce haut fait, qui témoigne de l'incroyable efficacité de ce mort, est hautement édifiant. D'abord parce qu'il rappelle que le pouvoir d'un palero dépend exclusivement du pouvoir du nfumbi; ensuite parce qu'il révèle que le palero n'est jamais aussi efficient que lorsqu'il est proche de son mort afin que celui-ci puisse venir spontanément à son secours. Chez Marelis, à en croire les commentaires de son entourage, ce degré de rapprochement atteint des sommets. Certains disent que la vieille palera s'est mariée avec son mort, d'autres qu'elle est devenue le mort lui-même. Si ce qu'on raconte sur Marelis atteste son pouvoir, ceci ne suffit pourtant pas à l'établir de manière durable. Les réputations se défont si elles ne sont pas actualisées. Nous verrons comment l'apparence de la palera et l'agencement de son cuarto sont un rappel constant de sa proximité avec les morts.

Les histoires véhiculées sur Marelis n'ont pas que des vertus anecdotiques car elles prédisposent le client à une certaine angoisse qui va conditionner son expérience de consultation et contribuer au changement attendu de son état. Cette angoisse grimpe d'un cran lorsqu'il la découvre dans son cuarto. Tout d'abord, il est intimidé par l'apparence même de Marelis. Assise à côté de son chaudron, la palera, avec sa forte corpulence, ses colliers rituels, ses yeux sombres et fixes, le cigare qu'elle n'arrête pas de mastiquer, incarne l'un des éléments les plus imposants du décor rituel.

Ensuite, le visiteur est déstabilisé car il pénètre dans un espace dont les éléments constitutifs lui sont étrangers et échappent à sa connaissance (Hanks 2009). Le cuarto palero, avec ses chaudrons mystérieux, les animaux disséqués, son obscurité, l'odeur de sang séché, est perturbant à plus d'un titre. Le cuarto de Marelis en fournit un exemple extrême. L'état de la pièce est saisissant avec des tables recouvertes de mèches de cheveux mélangées à la cire et aux rognures d'ongle. Sa saleté, avec des reliquats de rituels jonchant le sol et des restes d'animaux en putréfaction, sature les sens du visiteur et accentue le sentiment de malaise. Sur sa nganga, on peut voir souvent un pigeon vivant, immobile, alors qu'il n'est en rien entravé. Dans les placards, on entend des poulets crier, des tortues bouger ainsi que toutes sortes d'animaux qu'elle utilise pour les opérations. Tous ces bruits (mastication, grattements, cris, etc.), sorte de décor sonore, définissent aussi la construction de l'espace et de sa représentation. Comme le remarque le géographe Yi-Fu Tuan (1990), les sons produits dans un espace donné modifient non seulement la perception qu'une personne peut en avoir mais aussi son état émotionnel. Dans le cas de Marelis, l'inquiétude des visiteurs est renforcée par les bruits des animaux dont la plupart sont invisibles, cachés dans des boîtes, des placards et des tiroirs. Or un bruit est d'autant plus anxiogène que la source de son émission est inconnue. Selon Michael Rowlands, la matérialité du son (son volume, son intensité, la distance qui le sépare de son récepteur) fait partie du message communiqué lors d'une interaction. Cette matérialité crée des «enveloppes acoustiques», des bulles, qui donnent aux individus des clés pour comprendre dans quelle mesure ils sont capables de participer à l'interaction (Rowlands 2007). Les poulets qui crient dans les pla- 


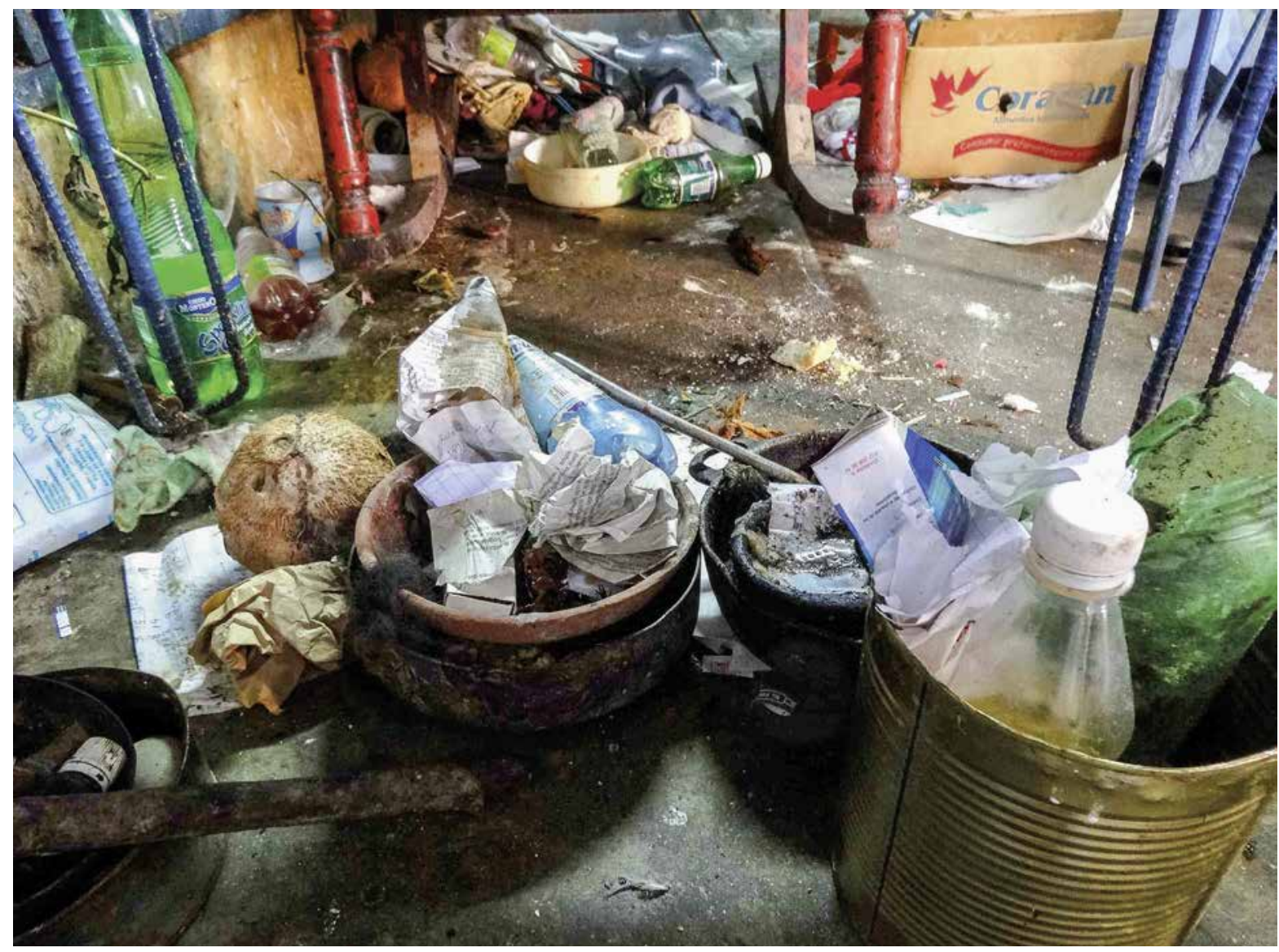

cards de Marelis ou le bruissement des ailes de pigeon derrière le chaudron créent autant de petites bulles sonores qui produisent elles-mêmes des petits espaces déplaisants, des espaces «négatifs» (Munn 1996: 448) dans lesquels le visiteur se sent étranger.

Marelis fait l'économie des artefacts spirituels. Seules sont visibles une nganga, l'effigie de bois d'un Amérindien et une «table de travail ». Pourtant le lieu produit une forte impression. Le cuarto de Marelis est exceptionnellement désordonné (fig. 10) faisant de la pièce dans laquelle elle reçoit ses clients une véritable énigme (y compris probablement pour elle-même, Marelis perdant une grande partie de son temps à chercher telle ou telle chose): l'espace est parsemé des composantes d'activités magiques en cours, d'innombrables bouts de papier où sont inscrites les conclusions des sessions divinatoires, de photos de ceux qui seront bientôt victimes de sa sorcellerie, de morceaux de bougie et de cigares jetés de-ci, de-là après utilisation. Sous la table, on aperçoit des cahiers surchargés de notes et plusieurs strates de livres entassés, dont le contenu varie, allant de la magie noire aux nouvelles technologies, en passant par la littérature. Derrière son fauteuil sont entreposés, en vrac, les cadeaux de ses clients, des poupées magiques, des pots remplis d'herbes médicinales. En fait, la pièce occupée par Marelis recèle un grand nombre de sous-espaces (subspaces) [Hanks 1996]: le dessous de la table en est un, l'arrière de son fauteuil en est un autre, l'intérieur d'un placard un troisième. Tous ces petits lieux ont pour effet de brouiller la capacité fig. 10

Chez Marelis, le désordre en-dessous de la table de travail. Cienfuegos, 2015. Photo Katerina Kerestetzi. 


\section{ci-contre}

fig. 11

Andrés. Cienfuegos, 2015.

Photo Katerina Kerestetzi. du visiteur à se représenter l'espace de manière synthétique. En effet, la prolifération d'éléments et de secteurs, la multi-localité, donnent l'impression d'un espace kaléidoscopique, de ce fait difficilement appréhendable (Tuan 1990). Le cuarto de Marelis est un espace prolifique dont les multiples facettes échappent à la perception du visiteur, créant d'emblée un fossé cognitif et par conséquent une dissymétrie interactionnelle entre le client et le praticien.

Si la manière dont Marelis a agencé son cuarto brouille la perception des visiteurs, ceci ne veut pas dire pour autant qu'il est, pour eux, dénué de sens. Au contraire, ces sous-espaces, qui peuvent prendre toutes les tailles et les formes, véhiculent des messages sur les puissances qui s'y cachent. II m'est ainsi arrivé d'ouvrir par hasard un livre de Gabriel García Márquez et d'y trouver inscrits des signes magiques, des occurrences divinatoires, des noms de clients. Cette habitude de marquer les livres (fig. 9) est presque une passion chez elle. Je me souviens qu'elle me demanda un jour de dessiner "l'architecture grecque» dans l'un de ses livres de magie. Comme il n'est pas conseillé de refuser quelque chose à Marelis, je me mis à griffonner un schéma approximatif du Parthénon et parvint à un résultat qui, je dois dire, était plutôt raté. Mais la vieille pratiquante en fut ravie car ce dessin, qui plus est de la main d'une Grecque, lui permettait désormais de disposer d'un peu de cette «puissance antique».

On doit mentionner ici le rôle des signes magiques, ou firmas, dans la perception de l'espace. De forme variable, les firmas sont constituées principalement de cercles, de flèches, de lignes et parfois de dessins figuratifs (Bonhomme et Kerestetzi 2015). II existe une pléthore de firmas pour représenter les morts, les divinités, les éléments naturels. Mais plus que de représenter, ces graphismes ont comme fonction de mobiliser l'agent figuré - en dessinant le symbole du tourbillon par exemple, on le fait «travailler» pour semer la confusion chez un ennemi. Ce qui importe ici est de savoir que lorsque la palera trace une firma sur une surface quelconque (une table, le sol, un livre), elle la transforme automatiquement en un locus d'agents spirituels. Lorsque Marelis crée des lieux de ce type, elle transforme des espaces virtuels (le Parthénon, le monde des morts) en espaces centrés et visibles; elle investit son espace immédiat des agents habituellement délocalisés; elle introduit le macrocosme, la demeure imaginée de ces agents, dans son microcosme, sa demeure réelle; elle fait de ces êtres abstraits, des entités agissantes (Hanks 1996). La puissance du Parthénon est en effet une notion abstraite. Mais lorsqu'on dessine le monument sur un livre d'un cuarto, c'est comme si on domestiquait son essence. Les frontières entre objet iconique et objet à trois dimensions se brouillent par leur parenté fonctionnelle.

Surcharger l'espace d'objets agissants, l'emplir de bruits inquiétants, combler chaque recoin d'objets étranges, cumuler des substances, rassembler des présences de toutes sortes, peut induire l'impression que Marelis officie dans un endroit vibrant, débordant jusqu'aux plis des nappes de puissances hétéroclites. Le client, pressentant que des entités étranges guettent partout dans la pièce, perçoit la dissymétrie interactionnelle comme l'expression d'une réelle asymétrie des rapports de pouvoir: lui est là tout seul alors que la palera est entourée d'une armée d'agents spirituels.

Et le hiatus se creuse davantage par le trouble que suscite la mise en contact de l'univers du palo avec des objets domestiques et personnels: un lit, un téléviseur, des vêtements, des journaux, un manomètre. 


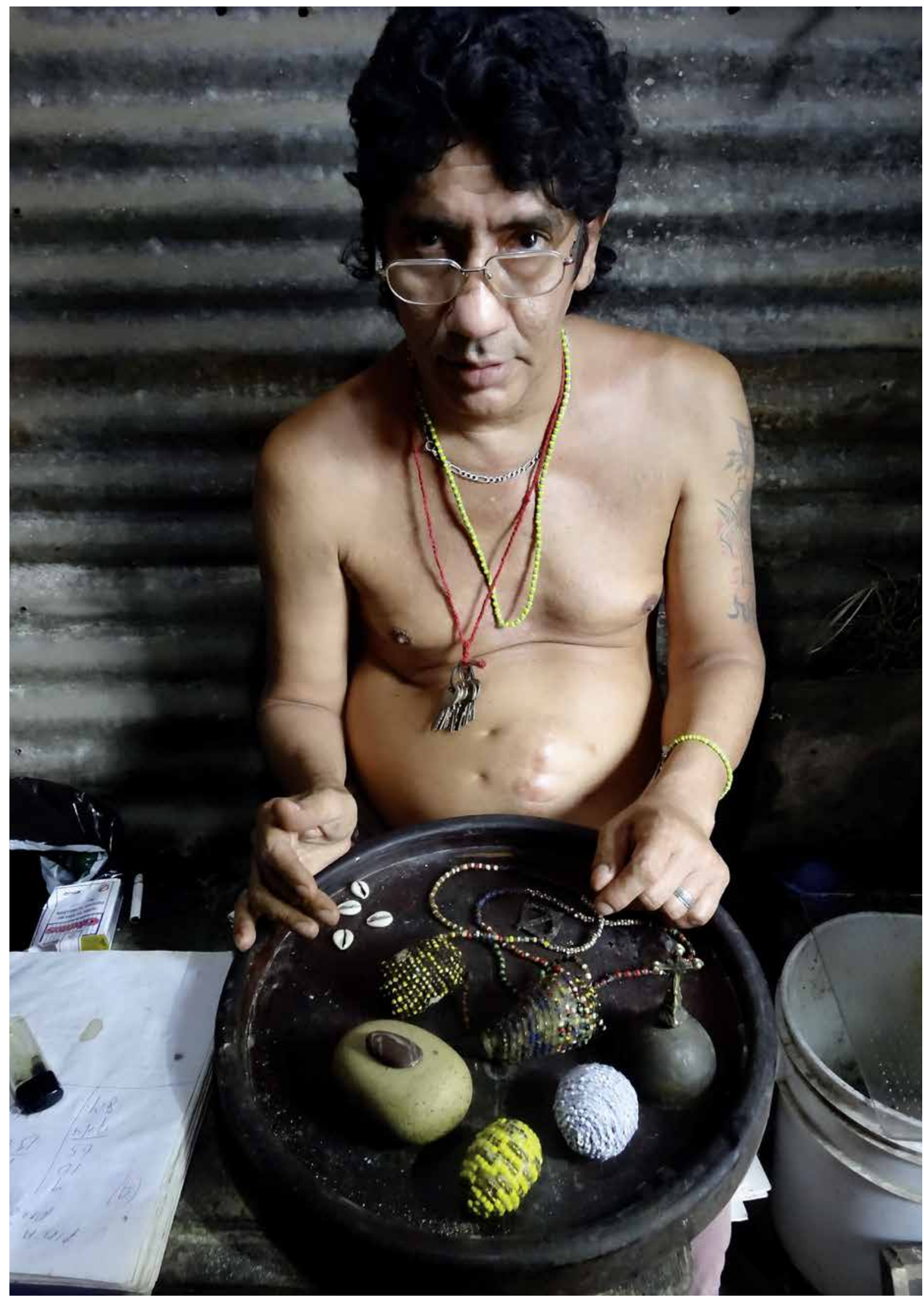




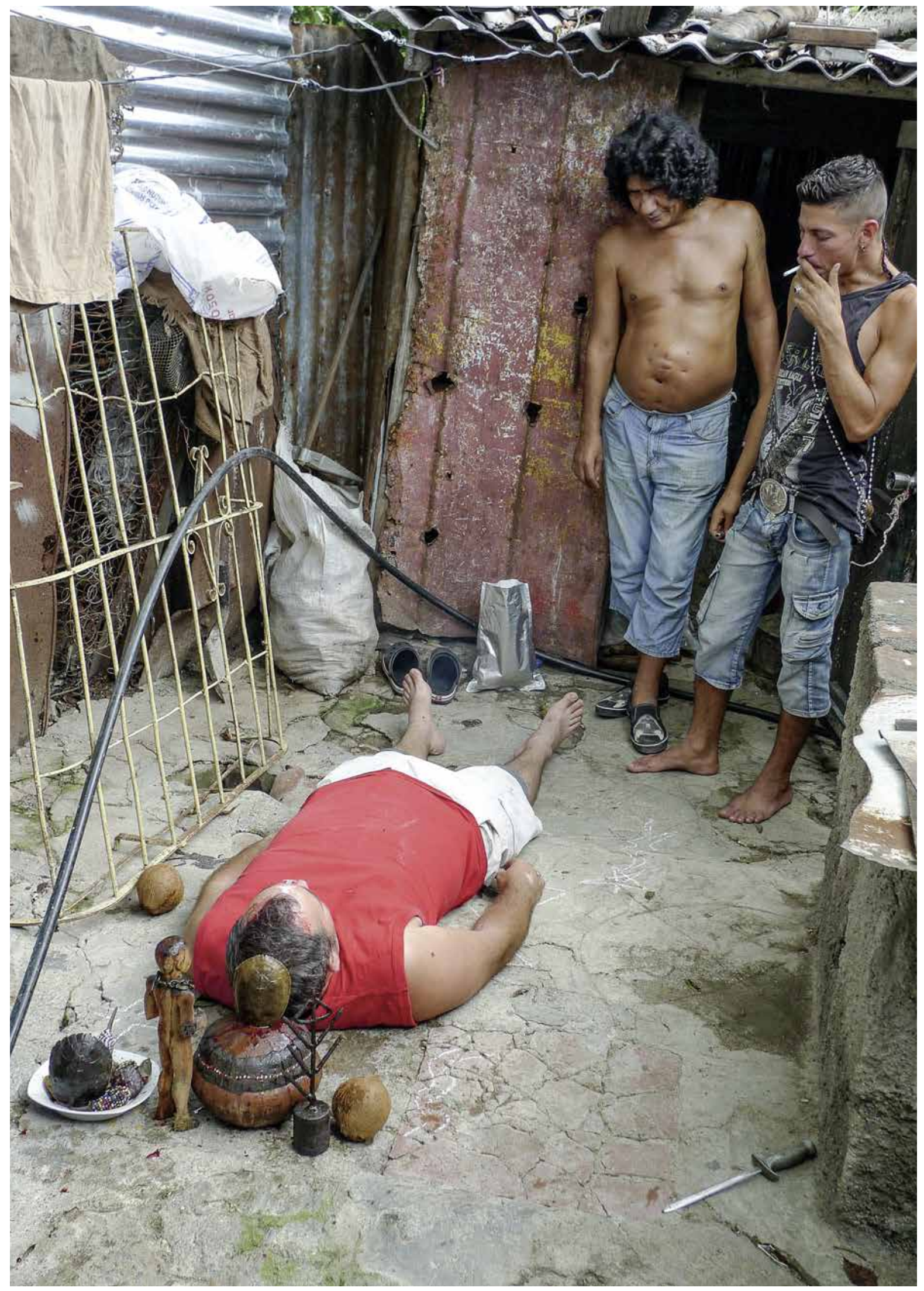


Marelis habite dans son sanctuaire. Il peut lui arriver ainsi d'interrompre un exorcisme pour mesurer sa pression sanguine ou pour déjeuner. L'imbrication de l'univers de l'extraordinaire et de la matérialité et des gestes de l'ordinaire, l'indifférenciation des registres de la ritualité et du quotidien, peuvent perturber le visiteur dont l'anxiété est comparable à ce sentiment dérangeant de out of place qu'engendre la disposition contre-intuitive de certains objets (Douglas 1981) ou le caractère déplacé de certains actes.

Mais c'est la proximité de la palera avec son mort qui frappe le plus. Marelis mange et dort aux côtés de sa nganga, sans précautions rituelles, sans barrières physiques. Le chant palero: «Là où vit ma nganga, je vis moi » (donde vive mi nganga, vivo yo), qu'elle chante invariablement lors de chaque rituel, dit vrai dans son cas. Et si elle peut le faire c'est qu'elle ne craint plus son mort; c'est lui qui vit dans sa chambre et c'est à lui qu'elle chuchote les derniers mots avant de s'endormir. Pour les visiteurs, cela ne fait aucun doute, Marelis vit avec les morts. On pourrait presque dire qu'elle a fusionné avec son cuarto ou qu'elle est une véritable composante de cet espace devenu nganga.

Cette proximité affichée avec son mort se retrouve dans l'agencement de la pièce. La nganga est placée dans un placard imbriqué dans le mur que Marelis ferme avec un cadenas les rares fois où elle quitte la pièce. Devant ce placard, elle a installé sa «table de travail», de sorte qu'elle est la seule à pouvoir accéder facilement à sa nganga. Les visiteurs sont maintenus à distance de cette zone de pouvoir, que nous appelons «épicentre d'action ", où se trouve installé le binôme palera/nganga. Le «statut de participation » (Goffman 1987) de chacun est ainsi défini spatialement, certains points dans l'espace ayant plus de ressources pour l'action que d'autres.

D'ailleurs, lorsqu'un assistant rituel quitte sa posture d'observateur, quand par exemple la chef lui ordonne d'asperger le chaudron avec du rhum, celui-ci doit se pencher vers l'avant, se serrer entre la table et Marelis, étendre son bras, étirer son cou... De l'autre côté, la palera peut agir dans une relative immobilité, en restant assise confortablement sur son grand fauteuil, avec sa nganga à portée de main.

Les clients doivent aussi se livrer à ces contorsions et ceci intervient dès leur toute première interaction avec la palera. Saluer Marelis oblige d'emblée à une soumission corporelle: son positionnement ainsi que la disposition des objets et des meubles vont forcément impacter leurs mouvements. S'il s'agit d'un proche - un initié, un client régulier, un ami qui vient demander conseil -, il lui faut pratiquement se pencher à quatre-vingt-dix degrés pour embrasser Marelis. Lorsque des personnes l'entourent ou que des objets sont déposés sur le sol, il devient si compliqué de l'atteindre que l'on se contente d'une salutation à distance. Marelis ordonne alors gentiment mais fermement au visiteur de s'exécuter malgré tout: «Hé, lindo ["mignon"] F., viens me faire un bisou! » De cette manière, elle invite le visiteur à réaliser une petite acrobatie dont le moment d'équilibre minimal est atteint lorsqu'elle le saisit par les épaules pour le gratifier d'un baiser. Ces premiers gestes forment donc une chorégraphie de l'inclinaison et du vacillement, une petite épreuve pour le visiteur qui lui permet déjà de gagner sa place dans la pièce. Petite épreuve, certes, source d'inconfort infime mais dont les effets cognitifs ne doivent pas être sous-estimés: les rapports de force entre les acteurs sont posés d'entrée de jeu. Peter Collett (cité par Duranti 1992 : 662) distingue les salutations symétriques, qui tendent à établir une solidarité entre individus égaux, des salutations asymétriques, qui ancrent dans le geste le respect vis-à-vis de

\section{ci-contre}

fig. 12

L'activité religieuse de la cour d'Andrés. Cienfuegos, 2012.

Photo Julien Bonhomme. 
personnes d'un statut supérieur. Marelis, qui n'a fait aucun effort pour saluer son visiteur, est clairement en position de domination alors que celui-ci éprouve la faiblesse du déséquilibre. L'inégalité est ainsi somatisée: l'autorité prend la forme d'une posture assise et relaxée et la déférence, celle d'un corps contorsionné et mal à l'aise. Marelis quitte rarement son siège et quand cela arrive, ses gestes ne sont ni théâtraux ni particulièrement dynamiques. À la différence d'autres paleros qui déploient des trésors d'énergie face à leur public, Marelis officie en toute économie, avec pour seuls atouts de grandes qualités d'élocution, sa longue expérience religieuse, son intelligence et sa vivacité d'esprit. Nul besoin de prouver son dévouement au palo, sa vie aux côtés de la nganga en est la démonstration la plus éclatante.

Chez son fils qui habite dans l'appartement adjacent, l'univers rituel est très différent. Même si Andrés possède une petite cour, il a préféré y bâtir son sanctuaire et séparer ainsi son activité liturgique de l'espace où il vit avec sa femme et son fils. Son cuarto est une cabane de bois très exiguë, de moins de deux mètres carrés (fig. 11). Celui qui vient consulter Andrés n'a d'autre choix que de s'installer bien en face de lui, à moins de quatre-vingts centimètres de distance et à la même hauteur. Cet agencement rend plus malaisée une consultation basée sur la parole - la distance est trop intime (Hall 1974) pour conférer aux discours du palero le poids nécessaire, d'autant plus qu'Andrés n'aime pas tant parler que de faire. À la différence de sa mère chez qui le geste est rare, Andrés déploie dans ses consultations une activité frénétique en mobilisant une multiplicité de supports dont la manipulation ne lui laisse aucun répit: il compulse ses cahiers religieux (les libretas), griffonne les résultats de ses divinations et confectionne des amulettes dont les innombrables ingrédients l'obligent à couper, gratter, tamiser, râper, pulvériser...

Andrés doit une part de cette variété gestuelle aux choix qu'il a fait pendant son apprentissage religieux. Même s'il a été formé au sein du même cercle de paleros que sa mère, il a également reçu l'enseignement de babalaos réputés et acquis certains secrets du culte d'Ifá qu'il a intégrés dans sa pratique du palo, dont certaines techniques de divination complexes, lui demandant une activité intellectuelle d'interprétation, de notation, de réflexion. Andrés a également obtenu de ces babalaos l'avatar d'Ossain, la divinité la plus sauvage de l'Ifá, de la forêt et des plantes, qui est même devenue son objet cultuel préféré. Or travailler avec Ossain, ce maître de la pharmacopée magique, exige de manipuler une impressionnante variété de plantes et de substances: dans une petite amulette, Andrés peut ainsi introduire jusqu'à quarante éléments différents.

Le client ne saurait comment contribuer à cette débauche de gestes qui échappe complètement à sa maîtrise. Chez Andrés, celui-ci n'est pourtant pas cantonné au rôle de simple spectateur, ce qui s'explique en partie par la configuration spatiale de la pièce. La distance qui sépare le client du palero est inférieure à un mètre, le premier étant donc par définition à proximité du centre de l'action. Andrés a en effet l'habitude d'impliquer certains de ses clients dans ce qu'il est en train de faire, que ce soit dans la fabrication des amulettes, la macération des herbes médicinales ou pour tenir un récipient... Si l'asymétrie entre la chef et ses timides visiteurs était évidente chez Marelis, chez Andrés, en revanche, la topographie du cuarto favorise le développement de relations plus symétriques fondées sur la participation et la coopération.

D'autres facteurs viennent renforcer ce sentiment de relative égalité entre le client et le praticien. Andrés cultive la proximité avec ses visiteurs en 
multipliant les blagues ou en parlant d'aspects pratiques de sa magie, ce qui a des vertus démystificatrices. Mais l'espace a encore une fois son rôle à jouer. Du point de vue du client, Andrés est au centre du champ de vision, entouré des innombrables pots de sa pharmacopée magique qui envahissent littéralement le lieu. Celui qui se trouve face à lui peut avoir l'impression d'être enfermé dans une armoire à pharmacie plutôt que dans un temple. Ce sentiment est renforcé par la relative discrétion des objets rituels du palero. La nganga n'est même pas dans le champ de vision du client. Rappelons que chez sa mère, quel que soit l'angle d'observation, Marelis est toujours associée visuellement à son chaudron, ce qui peut renforcer l'impression qu'il s'agit d'un couple inséparable et puissant. Chez Andrés, c'est la richesse de ses connaissances en botanique et en divination ainsi que son art dans les choses magiques qui sont étalés.

Chaque espace n'est pas fait pour n'importe quel type d'action, c'est une évidence. Du fait de l'étroitesse de son cuarto, Andrés délocalise ses consultations les plus gourmandes d'espace à l'extérieur, dans la cour plus spacieuse (fig. 12). La consultation palera comporte généralement deux phases qui équivalent à deux visites chez le pratiquant. Lors de la première visite, l'objectif est de déterminer, par la divination, l'origine des maux et le traitement adapté. Lors de la seconde, on applique ce traitement qui consiste d'habitude à purifier le patient par onction de sang et de rhum, à faire jaillir le feu..., tâches qui prennent de la place. Mais en déplaçant le rite dans la cour, le palero peut aussi faire intervenir son assistant rituel qui se trouve être son voisin d'en face. C'est à lui qu'Andrés va déléguer la partie manuelle du traitement. La cour donne directement accès à la maison d'Andrés et à l'entrée où se situe la file des clients. Tous, qu'ils soient voisins, parents, clients, sont donc témoins de ce qui se déroule là. D'une certaine manière, on pourrait dire que cette partie de la consultation est publique. D'“ ouvrier » de la magie qu'il était dans sa cabane, Andrés devient ici chef rituel à part entière. II supervise calmement et sûrement le déroulement de l'opération et dirige avec naturel les innombrables actions de son assistant sur le patient qui, dans une attitude de parfaite passivité, reçoit, sans mot dire, gorgées de rhum, sang et gerbes de feu. L'hyperactivité gestuelle a été conservée à l'extérieur du sanctuaire mais elle a été transmise à un tiers, l'assistant zélé. Alors qu'à l'intérieur du cuarto, elle mettait en valeur le savoir-faire d'Andrés, elle valorise ici son autorité.

\section{Conclusion}

Dans une veine cognitiviste, Whitehouse (2002) a émis l'idée de l'existence de modes divergents de religiosité, en partant du principe que le rituel, pour être transmis, doit laisser une trace durable dans la mémoire. II remarque que la mémoire à long terme des rituels est soit "sémantique" (connaissances générales, corpus théologiques), soit «épisodique» (biographique), selon la modalité de religiosité à laquelle on participe. Cet article reprend cette réflexion sous un autre angle, en proposant une approche qui part de l'esthétique de la pratique religieuse pour considérer les modalités d'une mémorisation somatique de la foi. Le corps s'entraîne pour une ritualité structurée autour de personnages, de performances ou d'artefacts, ainsi que par l'effort d'augmenter le son, de diriger le regard, par la concentration des odeurs, par l'orientation du mouvement. 
Pour étudier ces processus de mémorisation somatique, nous nous sommes intéressés non pas au corps considéré de manière isolée mais aux relations qu'entretiennent le corps et l'espace dans lequel il se meut. II s'agit d'un corps dans un espace, ou plutôt d'un espace considéré comme un corps externalisé, comme son miroir, le reflétant dans sa configuration, ses sensations et ses mouvements. Ce changement de focale analytique est une manière d'objectiver ce qui peut paraître de l'ordre du confidentiel et du privé, l'espace devenant une expression matérielle des états corporels.

La valeur heuristique de l'approche spatiale tient aussi au fait qu'elle articule sur un même niveau analytique la démarche sociologique - par définition l'espace réunit ou sépare - et cognitiviste - la sensorialité de l'espace étant information à part entière. En insistant sur le sens kinesthésique, nous proposons de considérer également à un même niveau les sensations et les idées - notre mouvement dans l'espace résultant d'une coordination de plusieurs organes et capteurs mais aussi de représentations et d'expériences d'actions passées (Berthoz 1997). Ainsi, manières de bouger et manières de penser s'imbriquent spatialement dans l'entrelacement des aspects matériels et symboliques de l'espace. En considérant enfin l'espace en tant que contenant, nous avons décrit les lieux confinés du palo monte et leur ambiance sensoriellement dense. Les effluves de sang frais et des animaux en décomposition, l'aspect mystérieux des morts incarnés, l'intensité des tambours et les moments de silence sont en effet tous amplifiés par l'étroitesse de l'espace faisant de la participation rituelle une expérience somatisée sensationnelle. Mais l'argument peut déborder largement le champ d'un culte domestique; il n'y a pas de religion qui échappe aux contraintes spatiales. Et chaque pratique religieuse occupe plusieurs places dans l'espace, certaines visibles et construites selon des normes réglementaires, d'autres personnalisées et confidentielles, mais toutes édifiantes. 


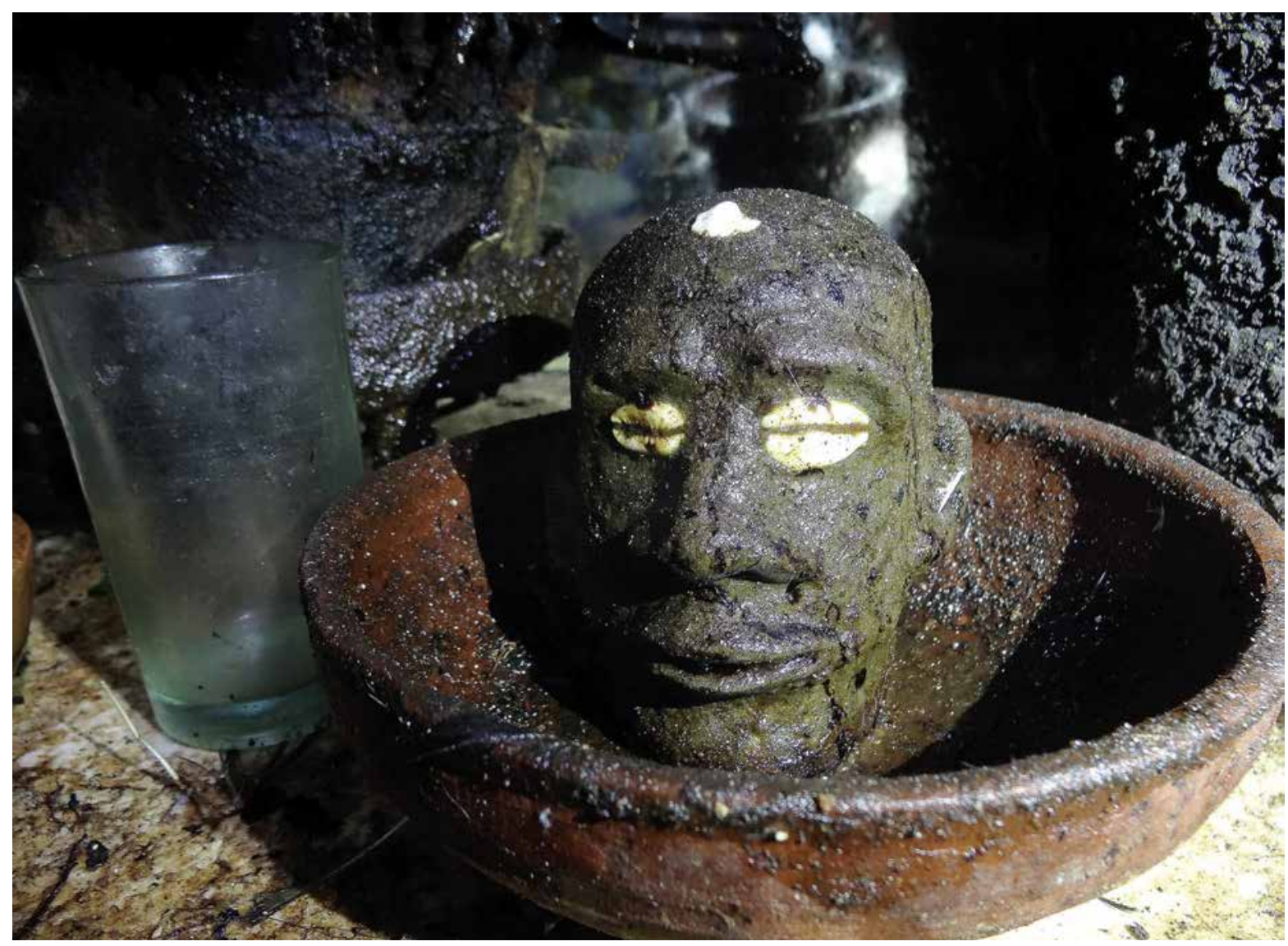

fig. 13

Lucero nourri de sang

animal. Cienfuegos, 2015.

Photo Katerina Kerestetzi. 


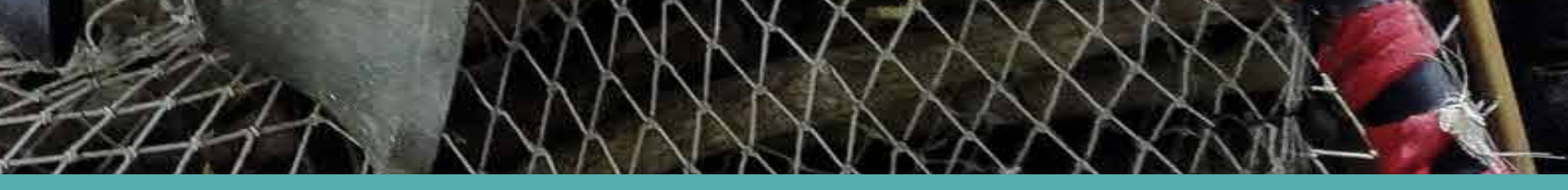

Bibliographie

Amerlinck, Mari-Jose

2001 «The Meaning and Scope of Architectural Anthropology ", in Mari-Jose Amerlinck (dir.) Architectural anthropology. Westport (États-Unis)/Londres Bergin et Garvey: 1-27.

\section{Argyriadis, Kali}

1999 La Religion à la Havane: actualité des représentations et des pratiques cultuelles havanaises. Paris, Archives contemporaines.

\section{Berthoz, Alain}

1997 Le Sens du mouvement. Paris, Odile Jacob.

\section{Bonhomme, Julien et Kerestetzi, Katerina}

2015 "Les signatures des dieux. Graphismes et action rituelle dans les religions afro-cubaines ",

Gradhiva 22

\section{Cabrera, Lydia}

$\mathbf{2 0 0 3}$ La Forêt et les dieux: religions afro-cubaines et médecine sacrée à Cuba, trad. de l'espagnol par Béatrice de Chavagnac. Paris, J.-M. Place.

\section{Castellanos, Jorge et Castellanos, Isabel}

1992 Cultura Afrocubana 3 : las religiones y las lenguas. Miami, Universal.

\section{Douglas, Mary}

1981 De la souillure: essai sur les notions de pollution et de tabou, trad. de l'anglais par Anne Guérin. Paris, La Découverte.

\section{Duranti, Alessandro}

1992 "Language and Bodies in Social Space: Samoan

Ceremonial Greetings », American Anthropologists 94 (3) : 657-691.

\section{Fernandez, James}

2003 «Emergence and Convergence in some African Sacred Places ", in Setha M. Low et Denise Lawrence-Zúñica (dir.), The Anthropology of Place and Space: Locating Culture. Oxford, Blackwell Publishing: 187-203.

\section{Gell, Alfred}

1998 Art and Agency: an Anthropological Theory. Oxford, Clarendon Press.

Architectures divines. Espace et kinesthésie dans le palo monte afro-cubain. Par Katerina Kerestetzi

Gibson, James J.

1977 "The Theory of Affordances», in Robert Shaw et John Bransford (dir.), Perceiving, Acting, and Knowing: Toward an Ecological Psychology. New York, Halsted Press Division, Wiley.

\section{Godard, Hubert}

1995 "Le geste et sa perception", in Isabelle Ginot et Marcelle Michel, La Danse au $x x^{e}$ siècle.

Paris, Larousse : 224-229.

\section{Goffman, Erving}

1987 Façons de parler, trad. de l'anglais par Alain Kinm. Paris, Les Éditions de Minuit.

\section{Hall, Edward T.}

1974 Handbook for Proxemic Research. Washington, Society for the Anthropology of Visual Communication.

\section{Halloy, Arnaud}

À paraître "L'odeur de l'axé. Pratiques olfactives et efficacité rituelle dans un culte afro-brésilien ", Journal de la Société des américanistes.

\section{Hanks, William}

1996 «Exorcism and the Description of Participant Roles », in Michael Silverstein et Greg Urban (dir.), Natural Histories of Discourse. Chicago, University of Chicago Press: 160: 202

2009 "Comment établir un terrain d'entente dans un rituel? ", Cahiers d'anthropologie sociale $5: 87-113$.

\section{Kerestetzi, Katerina}

2016 Vivre avec les morts à Cuba: réinvention et transmission religieuse dans le palo monte. Paris, Karthala.

\section{Lefebvre, Henri}

1974 La Production de l'espace. Paris, Anthropos.

Low, Setha M. et Lawrence-Zúñica, Denise

2003 "Locating Culture ", in Setha M. Low et Denise Lawrence-Zúñica (dir.), The Anthropology of Place and Space: Locating Culture. Oxford, Blackwell Publishing: 1-49.

\section{Munn, Nancy D.}

1996 «Excluded Spaces: the Figure in the Australian Aboriginal Landscape ",

Critical Inquiry 22 (3) : 446-465.

\section{Ochoa, Todd Ramón}

2010 Society of the Dead: Quita Manaquita and Palo Praise in Cuba. Berkeley, University of California Press.

\section{Ortiz, Fernando}

1906 Los negros brujos: apuntes para un estudio de etnología criminal. La Havane, Editorial de Ciencias Sociales.

\section{Pallasmaa, Juhani}

2005 The Eyes of the Skin: Architecture and the Senses. Chichester, Wiley-Academy.

\section{Palmié, Stephan}

2002 Wizards and Scientists :

Explorations in Afro-Cuban

Modernity and Tradition.

Durham, Duke University Press.

\section{Rapoport, Amos}

1982 The Meaning of the Built

Environment: A Nonverbal

Communication Approach

Beverly Hills, Sage Publications.

\section{Richardson, Miles}

2003 "Being-in-the-Market versus Being-in-the-Plaza: Material Culture and the Construction of Social Reality in Spanish America ", in Setha M. Low et Denise LawrenceZúñica (dir.), The Anthropology of Place and Space: Locating Culture. Oxford, Blackwell Publishing: 74-92.

\section{Rowlands, Michael}

2007 "The Sound of Witchcraft : Noise as Mediation in Religious Transmission ", in David Berliner et Ramon Sarró (dir.), Learning Religion: Anthropological Approaches. New York/Oxford, Berghahn Books.

\section{Tuan, Yi-Fu}

1990 "Space and Context ", in Richard Schechner et Willa Appel (dir.), By Means of Performance: Intercultural Studies of Theatre and Ritual. Cambridge/New York, Cambridge University Press : 236-245

\section{Warnier, Jean-Pierre}

2009 Régner au Cameroun: le roi-pot. Paris, Karthala.

\section{Whitehouse, Harvey}

2002 "Modes of Religiosity: Towards a Cognitive Explanation of the Sociopolitical Dynamics of Religion ", Method \& Theory in the Study of Religion 14 (3-4): 293-315. 


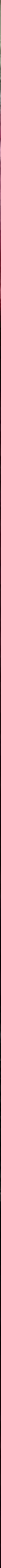

